\title{
Catalyst and Ring Size Effects on Periselectivity of Oxonium Ylide Rearrangements
}

\author{
Fredrik P. Marmsäter, John A. Vanecko and F. G. West* \\ Department of Chemistry, University of Alberta, W5-67 Gunning-Lemieux \\ Chemistry Centre, Edmonton, AB T6G 2G2, Canada, and \\ Department of Chemistry, University of Utah, 315 S. 1400 East, Rm 2020, Salt \\ Lake City, UT 84112
}

frederick.west@ualberta.ca

\section{Supporting Information}

\author{
Contents \\ Experimental Procedures.......................... S-1-S-7

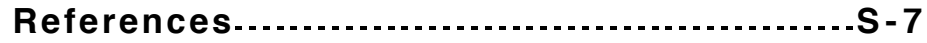 \\ NMR Spectra ................ S-8
}

General information. Reactions were carried out in flame-dried glassware under a positive nitrogen atmosphere unless otherwise stated. Transfer of anhydrous solvents and reagents was accomplished with oven-dried syringes or cannulae. Solvents were distilled before use: methylene chloride from calcium hydride, tetrahydrofuran and diethylether from sodium/benzophenone ketyl, toluene from sodium metal. Ethereal diazomethane was prepared from Diazald according to literature procedures. ${ }^{1}$ Thin layer chromatography was performed on glass plates precoated with $0.25 \mathrm{~mm}$ Kieselgel $60 \mathrm{~F}_{254}$ (Merck). Flash chromatography columns were packed with 230-400 mesh silica gel (Merck). Radial chromatography was carried out on a Chromatotron 7924T (Harrison Research) with plates prepared using silica gel 60 $\mathrm{F}_{254}$ with gypsum binder (EM) on glass rotors. Products isolated by chromatographic purification are listed in order of increasing polarity. Gas chromatograms were obtained on a Hewlett-Packard 5890 series II capillary gas chromatograph with a $30 \mathrm{~m} \mathrm{HP}-5$ column and a flame ionization detector interfaced with a Hewlett-Packard 3396 series II integrator. The following parameters were used: injection port 220 ${ }^{\circ} \mathrm{C}$, detector $250{ }^{\circ} \mathrm{C}$. Two different programs were used: (1) initial temperature of $140{ }^{\circ} \mathrm{C}$, held for $2 \mathrm{~min}$ then a linear temperature ramp of $10^{\circ} \mathrm{C} / \mathrm{min}$ to $300^{\circ} \mathrm{C}$, or (2) initial temperature of $120^{\circ} \mathrm{C}$, held for 2 min then a linear temperature ramp of $10{ }^{\circ} \mathrm{C} / \mathrm{min}$ to $300{ }^{\circ} \mathrm{C}$. Proton nuclear magnetic resonance spectra $\left({ }^{1} \mathrm{H}\right.$ NMR) were recorded at $300 \mathrm{MHz}, 400 \mathrm{MHz}$ or $500 \mathrm{MHz}$. The chemical shifts are reported on the $\square$ scale $(\mathrm{ppm})$ and the spectra are referenced to tetramethylsilane $\left(\square=0 \mathrm{ppm},{ }^{1} \mathrm{H} ;{ }^{13} \mathrm{C}\right)$ or to deuteriochloroform $\left(\square=7.26 \mathrm{ppm},{ }^{1} \mathrm{H} ; \square=77.23 \mathrm{ppm},{ }^{13} \mathrm{C}\right)$ as internal standard. Coupling constants $(J)$ are reported in Hertz $(\mathrm{Hz})$. Carbon nuclear magnetic resonance spectra $\left({ }^{13} \mathrm{C} \mathrm{NMR}\right)$ were recorded at 75 $\mathrm{MHz}, 100 \mathrm{MHz}$ or $125 \mathrm{MHz}$ and are reported (ppm) relative to the center line of the triplet from chloroform- $d(77.23 \mathrm{ppm})$. Infrared (IR) spectra were measured with a Mattson FTIR 3000 infrared spectrometer. Mass spectra were determined on a Finnigan Mat 95 high resolution gas chromatography/mass spectrometer with Finnigan Mat ICIS II operating system.

4,5-Bis(benzyloxy)-1-diazopentan-2-one 3a. To a solution of ethyl diazo acetate $(3.8 \mathrm{~mL}, 36$ $\mathrm{mmol})$ in dichloromethane $(72 \mathrm{~mL})$ was added $\mathrm{SnCl}_{2}(0.65 \mathrm{~g}, 3.4 \mathrm{mmol})$. The resulting bright yellow solution was mixed at r.t. for $15 \mathrm{~min}$ at which time the solution became cloudy. The reaction mixture was cooled to $-10{ }^{\circ} \mathrm{C}$ (acetone/ice bath) and a pre-formed solution of benzyloxyacetaldehyde (5.2 g, 34.0 $\mathrm{mmol})$ in dichloromethane $(19 \mathrm{~mL})$ was added dropwise via cannula. After the first few drops of the aldehyde solution, the resulting mixture began to bubble showing the evolution of nitrogen. Upon complete addition of the aldehyde solution, the reaction mixture was allowed to warm to r.t. and was 
stirred for and additional $10 \mathrm{~h}$. Water $(75 \mathrm{~mL})$ was added and the mixture was stirred for $15 \min$. The layers were separated and the organics were washed further with $\mathrm{H}_{2} \mathrm{O}(2 \times 50 \mathrm{~mL})$. The organic layer was then dried $\left(\mathrm{MgSO}_{4}\right)$ and concentrated to yield a yellow oil that was purified via flash column chromatography (silica gel; $4 \mathrm{~cm}$ x $10 \mathrm{~cm}$ column collecting $10 \mathrm{~mL}$ fractions; $5 \%$ EtOAc/hexanes) to yield $6.8 \mathrm{~g}(83 \%)$ of ethyl 4-benzyloxy-3-oxobutyrate as a colorless oil: $\mathrm{R}_{\mathrm{f}}=0.39(20 \%$ EtOAc/hexanes); ${ }^{1} \mathrm{H}$ NMR (300 $\left.\mathrm{MHz} \mathrm{CDCl}_{3}\right) \square 7.37-7.31(\mathrm{~m}, 5 \mathrm{H}), 4.59$ (s, 2H), 4.25-4.14 (m, 4H), $3.54(\mathrm{~s}, 2 \mathrm{H}), 1.25(\mathrm{t}, J=6.9 \mathrm{~Hz}, 3 \mathrm{H}) ;{ }^{13} \mathrm{C} \mathrm{NMR}\left(75 \mathrm{MHz} \mathrm{CDCl}_{3}\right) \square 202.0,167.2,137.1,128.8,128.7$, $128.1,75.0,73.7,61.6,46.3,14.3$. Data were consistent with literature values. ${ }^{2}$

The keto ester $(2.78 \mathrm{~g}, 11.8 \mathrm{mmol})$ was dissolved in EtOH $(41 \mathrm{~mL})$ and the solution was cooled to $-10{ }^{\circ} \mathrm{C}$. Solid $\mathrm{NaBH}_{4}(0.445 \mathrm{~g}, 11.8 \mathrm{mmol})$ was added in 4 portions over a period of $20 \mathrm{~min}$. The reaction was carefully monitored by TLC for the initial appearance of over-reduced product (baseline), after which it was quenched at $-10{ }^{\circ} \mathrm{C}$ by dropwise addition of $5 \% \mathrm{HCl}$. Ethyl acetate $(50 \mathrm{~mL})$ was added, and after vigorous stirring for $15 \mathrm{~min}$., the layers were separated. The aqueous layer was extracted with EtOAc $(50 \mathrm{~mL})$, and the combined organic layers were dried $\left(\mathrm{MgSO}_{4}\right)$ and concentrated to yield a yellow oil, which was purified by flash column chromatography (silica gel, $2 \mathrm{~cm} \mathrm{x} 10 \mathrm{~cm}$ column collecting $4 \mathrm{~mL}$ fractions; $200 \mathrm{~mL}$ each of 10\% EtOAc/hexanes and 20\% EtOAc/hexanes) to yield $1.94 \mathrm{~g}$ (70\%) of ethyl 4-benzyloxy-3-hydroxybutyrate as a colorless oil: ${ }^{1} \mathrm{H}$ NMR $\left(300 \mathrm{MHz}, \mathrm{CDCl}_{3}\right) \square 7.38$ $7.26(\mathrm{~m}, 5 \mathrm{H}), 4.56(\mathrm{~s}, 2 \mathrm{H}), 4.24$ (dddd, $J=10.8,10.8,6.2,4.6 \mathrm{~Hz}, 1 \mathrm{H}), 4.15(\mathrm{q}, J=7.2 \mathrm{~Hz}, 2 \mathrm{H}), 3.54-3.44$ $(\mathrm{m}, 2 \mathrm{H}), 3.04(\mathrm{~d}, J=4.4 \mathrm{~Hz}, 1 \mathrm{H}), 2.54(\mathrm{~d}, J=6.3 \mathrm{~Hz}, 1 \mathrm{H}), 1.26(\mathrm{t}, J=7.2 \mathrm{~Hz}, 3 \mathrm{H}) ;{ }^{13} \mathrm{C} \mathrm{NMR}(75 \mathrm{MHz}$, $\left.\mathrm{CDCl}_{3}\right) \square 172.4,138.0,128.6,128.0,128.0,73.6,73.3,67.4,60.9,38.4,14.4$. Data were consistent with literature values. ${ }^{2}$

Ethyl 4-benzyloxy-3-hydroxybutyrate $(0.171 \mathrm{~g}, 0.719 \mathrm{mmol})$ was dissolved in $\mathrm{PhH}(2.9 \mathrm{~mL})$ at r.t., and $\mathrm{Ag}_{2} \mathrm{O}(0.208 \mathrm{~g}, 0.898 \mathrm{mmol}), \mathrm{CaSO}_{4}(0.224 \mathrm{~g}, 1.62 \mathrm{mmol})$ and $\mathrm{PhCH}_{2} \mathrm{Br}(0.200 \mathrm{~mL}, 1.62 \mathrm{mmol})$ were then added sequentially. The reaction mixture was covered in aluminum foil and stirred for $3 \mathrm{~h}$ at r.t. Additional aliquots of $\mathrm{Ag}_{2} \mathrm{O}, \mathrm{CaSO}_{4}$ and $\mathrm{PhCH}_{2} \mathrm{Br}$ were then added (same quantities as before). And the reaction was stirred for an additional $8 \mathrm{~h}$, then filtered through Whatman \# 1 filter paper. The solid residue was washed with EtOAc $(50 \mathrm{~mL})$ and the combined organic layers were concentrated and purified by flash column chromatography (silica gel, $2 \mathrm{~cm}$ x $10 \mathrm{~cm}$ column collecting $10 \mathrm{~mL}$ fractions; $200 \mathrm{~mL}$ each of $5 \%$ EtOAc/hexanes and $15 \%$ EtOAc/hexanes) to yield $0.224 \mathrm{~g}$ (95\%) of ethyl 3,4bis(benzyloxy)butyrate as a colorless oil: $\mathrm{R}_{f}=0.44$ (30\% EtOAc/hexanes); IR (thin film) $2983,1750 \mathrm{~cm}$ ${ }^{1} ;{ }^{1} \mathrm{H}$ NMR $\left(300 \mathrm{MHz}, \mathrm{CDCl}_{3}\right) \square 7.37-7.23(\mathrm{~m}, 10 \mathrm{H}), 4.66\left(\mathrm{~d}, J_{\mathrm{AB}}=11.6 \mathrm{~Hz}, 1 \mathrm{H}\right), 4.62\left(\mathrm{~d}, J_{\mathrm{AB}}=11.6\right.$ $\mathrm{Hz}, 1 \mathrm{H}), 4.54(\mathrm{~s}, 2 \mathrm{H}), 4.17-4.06(\mathrm{~m}, 3 \mathrm{H}), 3.60(\mathrm{dd}, J=10.1,5.3 \mathrm{~Hz}, 1 \mathrm{H}), 3.53(\mathrm{dd}, J=10.1,5.3 \mathrm{~Hz}, 1 \mathrm{H})$, 2.63-2.60 (m, 2H), $1.23(\mathrm{t}, J=7.2 \mathrm{~Hz}, 3 \mathrm{H}) ;{ }^{13} \mathrm{C} \mathrm{NMR}\left(75 \mathrm{MHz}, \mathrm{CDCl}_{3}\right) \square$ 171.7, 138.6, 138.3, 128.6, $128.5,128.0,127.8,127.8,127.8,75.3,73.5,72.6,71.9,60.7,38.0,14.4$.

The ester $(0.512 \mathrm{~g}, 1.56 \mathrm{mmol})$ was dissolved in anhydrous $\mathrm{MeOH}(3 \mathrm{~mL})$ and an aqueous $2 \mathrm{~N}$ solution of $\mathrm{LiOH}(2.4 \mathrm{~mL}, 4.8 \mathrm{mmol})$ was added. The reaction mixture was stirred at r.t. for $5 \mathrm{~h}$, after which TLC showed complete consumption of starting ester. The reaction was extracted with EtOAc (15 $\mathrm{mL})$, and the aqueous layer was acidified with $6 \mathrm{~N} \mathrm{HCl}$, then extracted with EtOAc $(3 \times 20 \mathrm{~mL})$. The organic layers were dried $\left(\mathrm{MgSO}_{4}\right)$ and concentrated to yield an oil that was purified by flash column chromatography (silica gel, $2 \mathrm{~cm} \times 15 \mathrm{~cm}$ column collecting $8 \mathrm{~mL}$ fractions; $30 \% \mathrm{EtOAc/hexanes)} \mathrm{to} \mathrm{give}$ $0.426 \mathrm{~g} \mathrm{(91 \% )} \mathrm{of} \mathrm{3,4-bis(benzyloxy)butyric} \mathrm{acid} \mathbf{2 a}$ as a colorless oil: IR (thin film) 3158, 3088, 2865, $1711 \mathrm{~cm}^{-1}$; ${ }^{1} \mathrm{H}$ NMR $\left(500 \mathrm{MHz} \mathrm{CDCl}_{3}\right) \square 11.20$ (br s, $\left.1 \mathrm{H}\right), 7.38-7.27$ (m, $\left.10 \mathrm{H}\right), 4.69$ (d, $J_{\mathrm{AB}}=11.5 \mathrm{~Hz}$, $1 \mathrm{H}), 4.65\left(\mathrm{~d}, J_{\mathrm{AB}}=11.5 \mathrm{~Hz}, 1 \mathrm{H}\right), 4.57(\mathrm{~s}, 2 \mathrm{H}) 4.13-4.08(\mathrm{~m}, 1 \mathrm{H}), 3.63(\mathrm{dd}, J=10.1,5.1 \mathrm{~Hz}, 1 \mathrm{H}), 3.57(\mathrm{dd}$, $J=10.0,5.3 \mathrm{~Hz}, 1 \mathrm{H}), 2.75-2.66(\mathrm{~m}, 2 \mathrm{H}) ;{ }^{13} \mathrm{C} \mathrm{NMR}\left(125 \mathrm{MHz}, \mathrm{CDCl}_{3}\right) \square$ 177.4, 138.3, 138.1, 128.6, 128.5, 128.0, 127.9, 127.9, 127.8, 74.8, 73.6, 72.6, 71.5, 37.7; HRMS (FAB, M-H) for $\mathrm{C}_{18} \mathrm{H}_{19 \mathrm{O}} 4$ calcd $\mathrm{m} / \mathrm{z} 299.1283$, found $\mathrm{m} / \mathrm{z} 299.1256$.

Acid $\mathbf{2 a}$ was subjected to the general procedure given below for $\mathbf{2 b} \square \mathbf{3 b}$ : acid (838 $\mathrm{mg}, 2.79 \mathrm{mmol}$ ), $\mathrm{Et}_{2} \mathrm{O}(11.2 \mathrm{~mL}, 0.25 \mathrm{M}), \mathrm{Et}_{3} \mathrm{~N}(362 \mu \mathrm{L}, 2.79 \mathrm{mmol}, 1.0$ equiv), isobutylchloroformate $(389 \mu \mathrm{L}, 2.79$ mmol, 1.0 equiv), diazomethane $\left(\sim 16 \mathrm{mmol}\right.$, in $\sim 45 \mathrm{~mL}$ of $\left.\mathrm{Et}_{2} \mathrm{O}\right)$. The crude reaction mixture was purified by radial chromatography ( $4 \mathrm{~mm}$ plate, $300 \mathrm{~mL}$ each of $20 \%, 30 \%$ and $40 \%$ EtOAc/hexanes) to yield $683 \mathrm{mg}(76 \%)$ of diazoketone 3a as a yellow oil: $\mathrm{R}_{f} 0.18$ (30\% EtOAc/hexanes); IR (thin film) 3088, 2863, 2103, $1638 \mathrm{~cm}^{-1}$; ${ }^{1} \mathrm{H}$ NMR (500 MHz, $\left.\mathrm{CDCl}_{3}\right) \square 7.35-7.27$ (m, 10H), 5.26 (br s, 1H), 4.66 $\left(\mathrm{d}, J_{\mathrm{AB}}=11.6 \mathrm{~Hz}, 1 \mathrm{H}\right), 4.58\left(\mathrm{~d}, J_{\mathrm{AB}}=11.6 \mathrm{~Hz}, 1 \mathrm{H}\right), 4.56\left(\mathrm{~d}, J_{\mathrm{AB}}=12.1 \mathrm{~Hz}, 1 \mathrm{H}\right), 4.53\left(\mathrm{~d}, J_{\mathrm{AB}}=12.1 \mathrm{~Hz}\right.$, 1H) 4.13-4.11 (m, 1H), $3.57(\mathrm{~d}, J=4.8 \mathrm{~Hz}, 2 \mathrm{H}), 2.60$ (app br s, 2H); ${ }^{13} \mathrm{C} \mathrm{NMR}\left(125 \mathrm{MHz}, \mathrm{CDCl}_{3}\right) \square$ 
192.9, 138.5, 138.3, 128.7, 128.6, 128.5, 128.1, 127.9 (2), 75.4, 73.6, 72.7, 71.8, 55.7, 43.9 ; HRMS (CI, $\mathrm{M}+\mathrm{H}$ ) for $\mathrm{C}_{19} \mathrm{H}_{21} \mathrm{~N}_{2} \mathrm{O}_{3}$ calcd m/z 325.1552, found m/z 325.1551 .

4-Allyloxy-5-benzyloxy-1-diazopentan-2-one 3b. Ethyl 4-benzyloxy-3-hydroxybutyrate (0.109 g), $0.457 \mathrm{mmol}$ ) was treated as described above for the synthesis of ethyl 3,4-bis(benzyloxy)butyrate, except that allyl bromide ( 2 aliquots of $0.90 \mathrm{~mL} ; 1.03 \mathrm{mmol}$ ) were used in place of $\mathrm{PhCH}_{2} \mathrm{Br}$. The crude product was purified via flash column chromatography (silica gel, $2 \mathrm{~cm}$ x $18 \mathrm{~cm}$ column collecting $10 \mathrm{~mL}$ fractions; 5\% EtOAc/hexanes $(200 \mathrm{~mL})$ followed by $15 \% \mathrm{EtOAc} / \mathrm{hexanes})$ to yield $0.118 \mathrm{~g}(93 \%)$ of ethyl 3-allyloxy-4-benzyloxybutyrate as a colorless oil: $\mathrm{R}_{\mathrm{f}}=0.50$ (30\% EtOAc/hexanes); IR (thin film) 2982, 1735, $1651 \mathrm{~cm}^{-1}$; ${ }^{1} \mathrm{H}$ NMR $\left(300 \mathrm{MHz}, \mathrm{CDCl}_{3}\right) \square 7.37-7.25$ (m, 5H), 5.89 (ddt, $J=17.2,10.4,5.6$ $\mathrm{Hz}, 1 \mathrm{H}), 5.26$ (app dq, $J=17.2,1.6 \mathrm{~Hz}, 1 \mathrm{H}), 5.15$ (app dq, $J=10.3,1.3 \mathrm{~Hz}, 1 \mathrm{H}), 4.55$ (s, 2H), 4.16-3.98 $(\mathrm{m}, 5 \mathrm{H}), 3.57(\mathrm{dd}, J=10.1,5.2 \mathrm{~Hz}, 1 \mathrm{H}), 3.50(\mathrm{dd}, J=10.1,5.3 \mathrm{~Hz}, 1 \mathrm{H}), 2.62(\mathrm{dd}, J=15.7,5.7 \mathrm{~Hz}, 1 \mathrm{H})$, $2.55(\mathrm{dd}, J=15.7,7.1 \mathrm{~Hz}, 1 \mathrm{H}), 1.25(\mathrm{t}, J=7.1 \mathrm{~Hz}, 3 \mathrm{H}) ;{ }^{13} \mathrm{C} \mathrm{NMR}\left(75 \mathrm{MHz}, \mathrm{CDCl}_{3}\right) \square 171.7,138.3$, 135.1, 128.6, 127.8, 127.8, 117.1, 74.9, 73.5, 71.8, 71.5, 60.7, 37.9, 14.4; HRMS (C/I, M+H) calcd for $\mathrm{C}_{16} \mathrm{H}_{23} \mathrm{O}_{4} \mathrm{~m} / \mathrm{z} 279.1519$, found $\mathrm{m} / \mathrm{z} 279.1585$.

The ester $(0.706 \mathrm{~g}, 2.54 \mathrm{mmol})$ was treated with methanolic $\mathrm{LiOH}$ as described above for $\mathbf{2 a}$ and the crude product was purified by flash column chromatography (silica gel, 2 x $15 \mathrm{~cm}$ column collecting 8 $\mathrm{mL}$ fractions; $30 \%$ EtOAc/hexanes) to give $0.562 \mathrm{~g}(89 \%)$ of 3-allyloxy-4-benzyloxybutyric acid $\mathbf{2 b}$ as a colorless oil: $\mathrm{R}_{f}=0.29$ (30\% EtOAc/hexanes); IR (thin film) $3159,286,1714 \mathrm{~cm}^{-1} ;{ }^{1} \mathrm{H}$ NMR (300 MHz, $\left.\mathrm{CDCl}_{3}\right) \square 10.22$ (br s, 1H), 7.37-7.26 (m, 5H), $5.89(\mathrm{ddt}, J=17.2,10.3,5.7 \mathrm{~Hz}, 1 \mathrm{H}), 5.26(\operatorname{app~dq}, J=$ $17.3,1.6 \mathrm{~Hz}, 1 \mathrm{H}), 5.16(\operatorname{app~dq}, J=10.4,1.3 \mathrm{~Hz}, 1 \mathrm{H}), 4.56(\mathrm{~s}, 2 \mathrm{H}), 4.18-4.05(\mathrm{~m}, 2 \mathrm{H}), 4.00(\operatorname{app} \mathrm{dq}, J=$ 7.1, 5.2 Hz, 1H), $3.59(\mathrm{dd}, J=10.0,4.9 \mathrm{~Hz}, 1 \mathrm{H}), 3.52(\mathrm{dd}, J=10.0,5.3 \mathrm{~Hz}, 1 \mathrm{H}), 2.69(\mathrm{dd}, J=15.9,5.4$ $\mathrm{Hz}, 1 \mathrm{H}), 2.62(\mathrm{dd}, J=15.9,7.2 \mathrm{~Hz}, 1 \mathrm{H}) ;{ }^{13} \mathrm{C} \mathrm{NMR}\left(75 \mathrm{MHz}, \mathrm{CDCl}_{3}\right) \square 176.9,138.1,134.8,128.6$, 127.9, 127.9, 117.6, 74.5, 73.6, 71.5, 71.4, 37.6; HRMS (C/I, M+H) calcd for $\mathrm{C}_{14} \mathrm{H}_{19} \mathrm{O}_{4} \mathrm{~m} / \mathrm{z} 251.1205$, found $\mathrm{m} / \mathrm{z} 251.1266$.

To a solution of acid $2 \mathbf{b}(311 \mathrm{mg}, 1.24 \mathrm{mmol})$ in $\mathrm{Et}_{2} \mathrm{O}(5.0 \mathrm{~mL}, 0.25 \mathrm{M})$ cooled to $-10^{\circ} \mathrm{C}$ was added $\mathrm{Et}_{3} \mathrm{~N}(182 \mu \mathrm{L}, 1.31 \mathrm{mmol}, 1.05$ equiv) followed by isobutylchloroformate $(169 \mu \mathrm{L}, 1.31 \mathrm{mmol}, 1.05$ equiv). The reaction was stirred for $4 \mathrm{~h}$ and then filtered through a short pad of celite and added to a freshly prepared solution of diazomethane $\left(\sim 8 \mathrm{mmol}\right.$, in $\left.\sim 25 \mathrm{~mL}^{\circ} \mathrm{Et}_{2} \mathrm{O}\right)$ at $0{ }^{\circ} \mathrm{C}$ and stirred overnight. A stream of nitrogen was then introduced and the solvent was evaporated. The crude reaction mixture was purified by radial chromatography ( $4 \mathrm{~mm}$ plate, $200 \mathrm{~mL}$ each of $20 \%, 30 \%$ and $40 \%$ EtOAc/hexanes) to yield $224 \mathrm{mg}(66 \%)$ of diazoketone $\mathbf{3 b}$ as a yellow oil: $\mathrm{R}_{f} 0.21$ (30\% EtOAc/hexanes); IR (thin film) 3087, 2863, 2103, $1631 \mathrm{~cm}^{-1}$; ${ }^{1} \mathrm{H}$ NMR $\left(400 \mathrm{MHz} \mathrm{CDCl}_{3}\right) \square 7.37-7.27$ (m, 5H), 5.89 (dddd, $J=17.3$, $10.4,5.7,5.7 \mathrm{~Hz}, 1 \mathrm{H}), 5.30$ (br s, $1 \mathrm{H}), 5.25$ (dddd, $J=17.2,1.6,1.6,1.6 \mathrm{~Hz}, 1 \mathrm{H}), 5.15$ (dddd, $J=10.4$, $1.3,1.3,1.3 \mathrm{~Hz}, 1 \mathrm{H}), 4.57\left(\mathrm{~d}, J_{\mathrm{AB}}=12.1 \mathrm{~Hz}, 1 \mathrm{H}\right), 4.53\left(\mathrm{~d}, J_{\mathrm{AB}}=12.1 \mathrm{~Hz}, 1 \mathrm{H}\right), 4.14-4.00(\mathrm{~m}, 3 \mathrm{H}), 3.56-$ 3.49 (m, 2H), 2.57 (app br s, 2H); ${ }^{13} \mathrm{C}$ NMR (100 MHz, $\mathrm{CDCl}_{3}$ ) $\square$ 192.9, 138.3, 135.0, 128.6, 127.9 (2C), 117.2, 75.1, 73.5, 71.6, 71.4, 55.7, 43.8; HRMS (ES, M+Na) for $\mathrm{C}_{15} \mathrm{H}_{18} \mathrm{~N}_{2} \mathrm{O}_{3} \mathrm{Na}$ calcd m/z 297.1215, found $\mathrm{m} / \mathrm{z} 297.1215$.

5-Allyloxy-4-benzyloxy-1-diazopentan-2-one 3c. The procedure above for ethyl 4-benzyloxy-3oxobutyrate $\left[3.1 \mathrm{~mL}\right.$ ethyl diazoacetate $(29 \mathrm{mmol})$ and $0.52 \mathrm{~g} \mathrm{SnCl} \mathrm{S}_{2}(2.8 \mathrm{mmol})$ in $\mathrm{CH}_{2} \mathrm{Cl}_{2}$ ] was carried out using allyloxyacetaldehyde $(2.8 \mathrm{~g}, 28.0 \mathrm{mmol})$ in place of benzyloxyacetaldehyde. Purification by flash column chromatography (silica gel; $4 \mathrm{~cm}$ x $10 \mathrm{~cm}$ column collecting $10 \mathrm{~mL}$ fractions; $5 \%$ EtOAc/hexanes) yielded $6.8 \mathrm{~g}(83 \%)$ of ethyl 4-allyloxy-3-oxobutyrate as a colorless oil: $\mathrm{R}_{f}=0.39(20 \%$ EtOAc/hexanes); ${ }^{1} \mathrm{H}$ NMR $\left(300 \mathrm{MHz}, \mathrm{CDCl}_{3}\right) \square 5.96-5.82(\mathrm{~m}, 1 \mathrm{H}), 5.35-5.21(\mathrm{~m}, 2 \mathrm{H}), 4.31-4.16(\mathrm{~m}$, $3 \mathrm{H}), 4.13(\mathrm{~s}, 1 \mathrm{H}), 4.08-4.03(\mathrm{~m}, 2 \mathrm{H}) 3.54(\mathrm{~s}, 2 \mathrm{H}) 1.28(\mathrm{t}, J=7.2 \mathrm{~Hz}, 3 \mathrm{H}) ;{ }^{13} \mathrm{C} \mathrm{NMR}\left(75 \mathrm{MHz}, \mathrm{CDCl}_{3}\right) \square$ 202.1, 167.2, 133.7, 118.3, 75.0, 72.6, 61.6, 46.2, 14.3. Data were consistent with literature values. ${ }^{3}$

The keto ester $(333 \mathrm{mg}, 1.81 \mathrm{mmol})$ was dissolved in $\mathrm{EtOH}(7.3 \mathrm{~mL})$ and the reaction was cooled to $10^{\circ} \mathrm{C}$. To this was added $\mathrm{NaBH}_{4}(19 \mathrm{mg}, 0.49 \mathrm{mmol})$ and the reaction was stirred for $10 \mathrm{~min}$ and then quenched by the addition of $5 \mathrm{~mL}$ of $5 \% \mathrm{HCl}$ solution. The mixture was then extracted with $\mathrm{Et}_{2} \mathrm{O}(4 \times 20$ $\mathrm{mL}$ ), and the combined organic layers were washed with equal volumes of sat. $\mathrm{NaHCO}_{3}$, brine, then dried $\left(\mathrm{MgSO}_{4}\right)$, filtered and condensed under reduced pressure and purified by flash chromatography (silica gel, $3 \mathrm{~cm}$ x $15 \mathrm{~cm}$ column; $200 \mathrm{~mL}$ each of $25 \%$ and $35 \%$ EtOAc/hexanes) to yield $270 \mathrm{mg}(80 \%)$ of ethyl 4-allyloxy-3-hydroxybutyrate: $\mathrm{R}_{f} 0.38$ (50\% EtOAc/hexanes); IR $\left(\mathrm{CH}_{2} \mathrm{Cl}_{2}\right.$ cast) 3467, 3080, 1646, $1096 \mathrm{~cm}^{-1} ;{ }^{1} \mathrm{H}$ NMR $\left(300 \mathrm{MHz}, \mathrm{CDCl}_{3}\right.$ ) $\square 5.89$ (dddd, $\left.J=17.2,10.4,5.6,5.6 \mathrm{~Hz}, 1 \mathrm{H}\right), 5.27$ (dddd, $J=$ 
17.4, 1.7, 1.7, $1.7 \mathrm{~Hz}, 1 \mathrm{H}), 5.19$ (dddd, $J=10.4,1.3,1.3,1.3 \mathrm{~Hz}, 1 \mathrm{H}), 4.26-4.19(\mathrm{~m}, 1 \mathrm{H}), 4.17$ (q, $J=7.1$ $\mathrm{Hz}, 2 \mathrm{H}), 4.02(\mathrm{ddd}, J=5.6,1.5,1.5 \mathrm{~Hz}, 2 \mathrm{H}), 3.48(\mathrm{dd}, J=9.7,4.5 \mathrm{~Hz}, 1 \mathrm{H}), 3.43(\mathrm{dd}, J=9.7,6.0 \mathrm{~Hz}$, $1 \mathrm{H}), 2.98$ (app br d, $J=4.2 \mathrm{~Hz}, 1 \mathrm{H}), 2.53(\mathrm{~d}, J=6.2 \mathrm{~Hz}, 2 \mathrm{H}), 1.26(\mathrm{t}, J=7.1 \mathrm{~Hz}, 3 \mathrm{H}) ;{ }^{13} \mathrm{C}$ NMR $(75$ $\left.\mathrm{MHz}, \mathrm{CDCl}_{3}\right) \square 172.4,134.6,117.5,73.2,72.5,67.4,60.9,38.4,14.4$; HRMS (CI, $\left.\mathrm{M}+\mathrm{H}\right)$ for $\mathrm{C}_{9} \mathrm{H}_{17} \mathrm{O}_{4}$ calcd $\mathrm{m} / \mathrm{z}$ 189.1127, found: $\mathrm{m} / \mathrm{z} 189.1118$.

To a solution of the alcohol $(522 \mathrm{mg}, 2.78 \mathrm{mmol})$ in benzene $(11 \mathrm{~mL}, 0.25 \mathrm{M})$, in a flask covered with aluminum foil, was added $\mathrm{CaSO}_{4}$ (852 mg, $6.24 \mathrm{mmol}, 2.25$ equiv), $\mathrm{Ag}_{2} \mathrm{O}$ (805 mg, 3.47 mmol, 1.25 eq.) and benzyl bromide (742 $\mu \mathrm{L}, 6.24 \mathrm{mmol}, 2.25$ equiv) sequentially. The above additions were repeated at 2-hour intervals for a total of three additions. The reaction was then stirred for $12 \mathrm{~h}$, diluted with $\mathrm{CH}_{2} \mathrm{Cl}_{2}$ $(100 \mathrm{~mL})$ and filtered through celite, condensed and purified by flash chromatography (silica gel, $3 \mathrm{~cm} \mathrm{x}$ $19 \mathrm{~cm}$ column; $200 \mathrm{~mL}$ each of $10 \%$ and $20 \%$ EtOAc/hexanes) to yield $747 \mathrm{mg}(97 \%)$ of ethyl 4allyloxy-3-benzyloxy butyrate: $\mathrm{R}_{f} 0.53$ (30\% EtOAc/hexanes); IR (thin film) 3012, 2990, $1732 \mathrm{~cm}^{-1} ;{ }^{1} \mathrm{H}$ NMR (300 MHz, $\left.\mathrm{CDCl}_{3}\right)$; 7 7.34-7.26 (m, 5H), 5.96-5.83 (m, 1H), 5.31-5.16 (m, 2H), $4.68\left(\mathrm{~d}, J_{\mathrm{AB}}=\right.$ $11.6 \mathrm{~Hz}, 1 \mathrm{H}), 4.62\left(\mathrm{~d}, J_{\mathrm{AB}}=11.6 \mathrm{~Hz}, 1 \mathrm{H}\right), 4.13(\mathrm{qd}, J=7.1,1.6 \mathrm{~Hz}, 2 \mathrm{H}), 4.10-4.09(\mathrm{~m}, 1 \mathrm{H}), 4.01$ (app dt, $J=5.5,1.3 \mathrm{~Hz}, 2 \mathrm{H}), 3.57(\mathrm{dd}, J=10.1,5.3 \mathrm{~Hz}, 1 \mathrm{H}), 3.50(\mathrm{dd}, J=10.1,5.3 \mathrm{~Hz}, 1 \mathrm{H}), 2.62-2.59(\mathrm{~m}, 2 \mathrm{H})$, $2.44(\mathrm{t}, J=7.0 \mathrm{~Hz}, 3 \mathrm{H}) ;{ }^{13} \mathrm{C}$ NMR $\left(75 \mathrm{MHz}, \mathrm{CDCl}_{3}\right) \square 171.7,138.6,134.8,128.5,128.0,127.8,117.2$, 75.2, 72.7, 72.5, 71.9, 60.7, 38.0, 14.4; HRMS (CI, M+H) for $\mathrm{C}_{16} \mathrm{H}_{23} \mathrm{O}_{4}$ calcd $\mathrm{m} / \mathrm{z} 279.1596$, found $\mathrm{m} / \mathrm{z}$ 279.1584 .

To a solution of ethyl 4-allyloxy-3-benzyloxy butyrate $(860 \mathrm{mg}, 3.1 \mathrm{mmol})$ in $\mathrm{MeOH}(6.2 \mathrm{~mL})$ was added $3.1 \mathrm{~mL}$ of $2 \mathrm{~N} \mathrm{LiOH}$. The reaction was stirred until completion as ascertained by TLC ( $3 \mathrm{~h})$ and the solution was transferred to a separatory funnel and $5 \mathrm{~mL}$ of $1 \mathrm{~N} \mathrm{NaOH}$ was added. The aqueous phase was then washed with $20 \mathrm{~mL}$ of EtOAc, acidified to $\mathrm{pH} 1 \sim 2$ with $3 \mathrm{~N} \mathrm{HCl}$ and extracted with EtOAc $(3 \times 20 \mathrm{~mL})$. The combined organic layers were dried $\left(\mathrm{MgSO}_{4}\right)$ and condensed to afford 4allyloxy-3-benzyloxybutyric acid $2 \mathrm{c}$ as a dark yellow oil $(730 \mathrm{mg}, 95 \%)$ which was taken on without further purification.

Crude acid $\mathbf{2 c}$ was subjected to the general procedure described above for $\mathbf{2} \mathbf{b} \square \mathbf{3 b}$ : acid (270 $\mathrm{mg}$, $1.08 \mathrm{mmol}), \mathrm{Et}_{2} \mathrm{O}(4.3 \mathrm{~mL}, 0.25 \mathrm{M}), \mathrm{Et}_{3} \mathrm{~N}(158 \mu \mathrm{L}, 1.13 \mathrm{mmol}, 1.05$ equiv), isobutylchloroformate (147 $\mu \mathrm{L}, 1.13$ mmol, 1.05 equiv), diazomethane $\left(\sim 8 \mathrm{mmol}\right.$, in $\left.\sim 25 \mathrm{~mL}_{\text {of }} \mathrm{Et}{ }_{2} \mathrm{O}\right)$. The crude reaction mixture was purified by radial chromatography ( $4 \mathrm{~mm}$ plate, $200 \mathrm{~mL}$ each of $15 \%, 25 \%$ and $40 \% \mathrm{EtOAc} / \mathrm{hexanes}$ ) to yield $200 \mathrm{mg}(69 \%)$ of diazoketone 3c as a yellow oil: $\mathrm{R}_{f} 0.19$ (30\% EtOAc/hexanes); IR $\left(\mathrm{CH}_{2} \mathrm{Cl}_{2}\right.$ cast) $3030,2862,2103,1639,1366,1096 \mathrm{~cm}^{-1} ;{ }^{1} \mathrm{H}$ NMR (300 MHz, $\left.\mathrm{CDCl}_{3}\right) \square 7.35-7.25(\mathrm{~m}, 5 \mathrm{H}), 5.96-$ $5.83(\mathrm{~m}, 1 \mathrm{H}), 5.31-5.16(\mathrm{~m}, 3 \mathrm{H}), 4.67\left(\mathrm{~d}, J_{A B}=11.6 \mathrm{~Hz}, 1 \mathrm{H}\right), 4.58\left(\mathrm{~d}, J_{A B}=11.6 \mathrm{~Hz}, 1 \mathrm{H}\right), 4.13-4.06(\mathrm{~m}$, $1 \mathrm{H}), 4.02-3.99(\mathrm{~m}, 2 \mathrm{H}) 3.53(\mathrm{~d}, J=4.9 \mathrm{~Hz}, 2 \mathrm{H}), 2.59-2.58(\operatorname{app}$ br s, $2 \mathrm{H}) ;{ }^{13} \mathrm{C} \mathrm{NMR}\left(75 \mathrm{MHz}, \mathrm{CDCl}_{3}\right)$ $\square 192.9,138.5,134.7,128.5,128.0,127.9,117.3,75.3,72.7,72.5,71.8,55.7,43.8$; HRMS (ES, M+Na) for $\mathrm{C}_{15} \mathrm{H}_{18} \mathrm{~N}_{2} \mathrm{O}_{3} \mathrm{Na}$ calcd m/z 297.1215, found $\mathrm{m} / \mathrm{z} 297.1215$.

General Procedure for Catalytic Carbene Transfer Reactions of Diazoketones using Rhodium Catalysts. To a mixture of the solvent $(0.01 \mathrm{M})$ and catalyst was added dropwise via cannula or syringe a solution of the diazoketone $(0.1 \mathrm{M})$. When the addition was complete the reaction was monitored by TLC and concentrated upon consumption of the diazoketone. The crude reaction mixture was passed through a short silica gel pad, eluting with 50\% EtOAc/hexanes, analyzed by GC, then purified by radial or flash chromatography.

General Procedure for Catalytic Carbene Transfer Reactions of Diazoketones using Copper Catalysts. To a mixture of the solvent $(0.01 \mathrm{M})$ and catalyst was added dropwise via cannula or syringe a solution of the diazoketone $\left(0.1 \mathrm{M}\right.$ in $\left.\mathrm{CH}_{2} \mathrm{Cl}_{2}\right)$. When the addition was complete the reaction was monitored by TLC and upon consumption of the diazoketone $0.5 \mathrm{M} \mathrm{K}_{2} \mathrm{CO}_{3}$ was added. The aqueous phase was then extracted with $\mathrm{CH}_{2} \mathrm{Cl}_{2}(2 \mathrm{x})$ and the combined organic phase was dried with $\mathrm{MgSO}_{4}$, filtered, and condensed. The crude reaction mixture was passed through a short silica gel pad, eluting with $50 \%$ EtOAc/hexanes, analyzed by GC, then purified by radial or flash chromatography.

Decomposition of 4,5-Bis(benzyloxy)-1-diazopentan-2-one 3a with $\mathbf{R h}_{2}(\mathbf{t p a})_{4}$. Diazoketone 3a (40 mg, $0.12 \mathrm{mmol})$ was subjected to the general procedure $\left[\mathrm{Rh}_{2}(\mathrm{tpa})_{4}(2 \mathrm{mg}, 0.0024 \mathrm{mmol}), \mathrm{CH}_{2} \mathrm{Cl}_{2}\right.$ $(12 \mathrm{~mL}, 0.01 \mathrm{M})$ ] followed by radial chromatography $(2 \mathrm{~mm}$ plate, $50 \mathrm{~mL}$ hexanes, $100 \mathrm{~mL}$ each of $7.5 \%$, $15 \%$ and $25 \% \mathrm{EtOAc} /$ hexanes) to yield $4.3 \mathrm{mg}$ (12\%) of $\mathbf{5 a}$ (major diast.), $14 \mathrm{mg}$ (38\%) of $\mathbf{4 a}$ (major 
diast.), $7.3 \mathrm{mg}(21 \%)$ of $\mathbf{4 a}$ (minor diast.) and $1 \mathrm{mg}(3 \%)$ of a minor product tentatively assigned as the other diastereomer of pyran $\mathbf{5 a}$.

Decomposition of 4-Benzyloxy-5-benzyloxy-1-diazopentan-2-one 3a with $\mathrm{Cu}(\mathrm{tfacac})_{2}$. Diazoketone 3a $(53 \mathrm{mg}, 0.16 \mathrm{mmol})$ was subjected to the general procedure [Cu(tfacac) $(5 \mathrm{mg}, 0.016$ $\mathrm{mmol}), \mathrm{CH}_{2} \mathrm{Cl}_{2}(15 \mathrm{~mL}, 0.01 \mathrm{M})$ ] followed by radial chromatography $(2 \mathrm{~mm}$ plate, $100 \mathrm{~mL}$ hexanes, 100 $\mathrm{mL}$ each of $5 \%, 10 \%, 15 \%$, and 30\% EtOAc/hexanes) to yield $2 \mathrm{mg}(4 \%)$ of $\mathbf{5 a}$ (major diast.), $19 \mathrm{mg}$ $(40 \%)$ of $\mathbf{6 a}, 10 \mathrm{mg}(21 \%)$ of $\mathbf{4 a}$ (major diast.), $6 \mathrm{mg}(12 \%)$ of $\mathbf{4 a}$ (minor diast.) and $1 \mathrm{mg}(2 \%)$ of a minor product tentatively assigned as the other diastereomer of pyran $\mathbf{5 a}$.

Enol ether 6a: $\mathrm{R}_{f} 0.50$ (30\% EtOAc/hexanes); ${ }^{1} \mathrm{H}$ NMR (500 MHz, $\left.\mathrm{CDCl}_{3}\right) \square$ 7.37-7.29 (m, 10H), $5.98(\mathrm{dd}, J=2.0,2.0 \mathrm{~Hz}, 1 \mathrm{H}), 4.71-4.66(\mathrm{~m}, 1 \mathrm{H}), 4.70(\mathrm{~s}, 2 \mathrm{H}), 4.63\left(\mathrm{~d}, J_{\mathrm{AB}}=12.2 \mathrm{~Hz}, 1 \mathrm{H}\right), 4.60\left(\mathrm{~d}, J_{\mathrm{AB}}=\right.$ $12.2 \mathrm{~Hz}, 1 \mathrm{H}), 3.63(\mathrm{dd}, J=10.3,6.5 \mathrm{~Hz}, 1 \mathrm{H}), 3.53(\mathrm{dd}, J=10.3,4.4 \mathrm{~Hz}, 1 \mathrm{H}), 2.84$ (ddd, $J=14.7,10.3$, $2.0 \mathrm{~Hz}, 1 \mathrm{H}), 2.63(\mathrm{ddd}, J=14.7,7.8,2.0 \mathrm{~Hz}, 1 \mathrm{H}) ;{ }^{13} \mathrm{C} \mathrm{NMR}\left(125 \mathrm{MHz}, \mathrm{CDCl}_{3}\right) \square 141.5,138.3,136.8$, 129.0, 128.7, 128.6, 128.3, 127.9, 127.9, 121.5, 78.1, 73.6, 72.5, 72.3, 33.3; HRMS (FAB, m- $\left.\mathrm{C}_{7} \mathrm{H}_{8}\right)$ for $\mathrm{C}_{12} \mathrm{H}_{12} \mathrm{O}_{3}$ calcd m/z 204.0786 found $\mathrm{m} / \mathrm{z} .204 .0790$.

2-Benzyl-5-benzyloxytetrahydropyran-3-one 5a (major): $\mathrm{R}_{f} 0.48$ (30\% EtOAc/hexanes); $\mathrm{R}_{t}$ $16.4 \mathrm{~min}$; IR $\left(\mathrm{CH}_{2} \mathrm{Cl}_{2}\right.$ cast) 3031, 2930, $1715 \mathrm{~cm}^{-1} ;{ }^{1} \mathrm{H}$ NMR (500 $\left.\mathrm{MHz}, \mathrm{CDCl}_{3}\right) \square ; 7.34-7.19(\mathrm{~m}, 10 \mathrm{H})$, $4.56\left(\mathrm{~d}, J_{\mathrm{AB}}=11.9 \mathrm{~Hz}, 1 \mathrm{H}\right), 4.47\left(\mathrm{~d}, J_{\mathrm{AB}}=11.9 \mathrm{~Hz}, 1 \mathrm{H}\right), 4.20(\mathrm{dd}, J=11.9,5.7 \mathrm{~Hz}, 1 \mathrm{H}), 4.07-4.02(\mathrm{~m}$, $1 \mathrm{H}), 4.00(\mathrm{dd}, J=8.7,3.5 \mathrm{~Hz}, 1 \mathrm{H}), 3.59(\mathrm{dd}, J=11.9,6.1 \mathrm{~Hz}, 1 \mathrm{H}), 3.21(\mathrm{dd}, J=14.6,3.5 \mathrm{~Hz}, 1 \mathrm{H}), 2.83-$ $2.77(\mathrm{~m}, 2 \mathrm{H}), 2.61(\mathrm{dd}, J=15.2,6.6 \mathrm{~Hz}, 1 \mathrm{H}) ;{ }^{13} \mathrm{C} \mathrm{NMR}\left(125 \mathrm{MHz}, \mathrm{CDCl}_{3}\right) \square 207.8,138.0,137.8,129.6$, $128.7,128.5,128.1,127.9,126.7,83.2,73.4,71.1,69.8,43.3,36.2$; HRMS (CI) for $\mathrm{C}_{19} \mathrm{H}_{20} \mathrm{O}_{3}$ calcd 296.1412, found: $\mathrm{m} / \mathrm{z} 296.1408$.

2-Benzyl-5-(benzyloxymethyl)tetrahydrofuran-3-one $\quad 4 a \quad$ (major): $\mathrm{R}_{f} \quad 0.42 \quad(30 \%$ EtOAc/hexanes); $\mathrm{R}_{t} 15.8 \mathrm{~min}$; IR $\left(\mathrm{CH}_{2} \mathrm{Cl}_{2}\right.$ cast) $3031,1740 \mathrm{~cm}^{-1} ;{ }^{1} \mathrm{H}$ NMR $\left(500 \mathrm{MHz}, \mathrm{CDCl}_{3}\right)$ 7.35$7.21(\mathrm{~m}, 10 \mathrm{H}), 4.56\left(\mathrm{~d}, J_{\mathrm{AB}}=12.3 \mathrm{~Hz}, 1 \mathrm{H}\right), 4.50\left(\mathrm{~d}, J_{\mathrm{AB}}=12.2 \mathrm{~Hz}, 1 \mathrm{H}\right), 4.37-4.34(\mathrm{~m}, 2 \mathrm{H}), 3.66(\mathrm{dd}, J=$ $10.2,3.4 \mathrm{~Hz}, 1 \mathrm{H}), 3.49(\mathrm{dd}, J=10.3,3.8 \mathrm{~Hz}, 1 \mathrm{H}), 3.03(\mathrm{dd}, J=14.3,4.3 \mathrm{~Hz}, 1 \mathrm{H}), 2.86(\mathrm{dd}, J=14.3,6.8$ $\mathrm{Hz}, 1 \mathrm{H}), 2.41(\mathrm{ddd}, J=18.0,4.6,0.6 \mathrm{~Hz}, 1 \mathrm{H}), 2.23(\mathrm{dd}, J=18.0,8.4 \mathrm{~Hz}, 1 \mathrm{H}) ;{ }^{13} \mathrm{C}$ NMR $(125 \mathrm{MHz}$, $\left.\mathrm{CDCl}_{3}\right) \square 215.8,138.0,137.1,129.9,128.7,128.6,127.9,127.7,126.8,80.8,74.7,73.6,73.0,39.0,38.0$; HRMS (CI) for $\mathrm{C}_{19} \mathrm{H}_{20} \mathrm{O}_{3}$ calcd m/z 296.1412, found $\mathrm{m} / \mathrm{z} 296.1403$.

2-Benzyl-5-(benzyloxymethyl)tetrahydrofuran-3-one $\quad 4 a \quad$ (minor): $R_{f} \quad 0.38 \quad(30 \%$ EtOAc/hexanes); $\mathrm{R}_{t} 15.8 \mathrm{~min}$; IR (thin film) 3037, 2862, $1758 \mathrm{~cm}^{-1} ;{ }^{1} \mathrm{H} \mathrm{NMR}\left(500 \mathrm{MHz}, \mathrm{CDCl}_{3}\right.$ ) $7.39-7.18(\mathrm{~m}, 10 \mathrm{H}), 4.60\left(\mathrm{~d}, J_{\mathrm{AB}}=12.2 \mathrm{~Hz}, 1 \mathrm{H}\right), 4.57\left(\mathrm{~d}, J_{\mathrm{AB}}=12.5 \mathrm{~Hz}, 1 \mathrm{H}\right), 4.39-4.34(\mathrm{~m}, 1 \mathrm{H}), 4.10(\mathrm{dd}$, $J=7.0,3.8 \mathrm{~Hz}, 1 \mathrm{H}), 3.58(\mathrm{dd}, J=10.5,3.8 \mathrm{~Hz}, 1 \mathrm{H}), 3.54(\mathrm{dd}, J=10.5,5.1 \mathrm{~Hz}, 1 \mathrm{H}), 3.09(\mathrm{dd}, J=14.5$, $4.0 \mathrm{~Hz}, 1 \mathrm{H}), 2.91(\mathrm{dd}, J=14.3,7.0 \mathrm{~Hz}, 1 \mathrm{H}), 2.46(\mathrm{dd}, J=18.2,6.3 \mathrm{~Hz}, 1 \mathrm{H}), 2.13$ (dd, $J=18.1,9.9 \mathrm{~Hz}$, $1 \mathrm{H}) ;{ }^{13} \mathrm{C}$ NMR $\left(125 \mathrm{MHz}, \mathrm{CDCl}_{3}\right) \square 215.0,138.1,137.3,129.9,128.7,128.4,128.0,127.9,126.8,82.1$, 75.2, 73.7, 71.8, 39.8, 37.5; HRMS (EI) for $\mathrm{C}_{19} \mathrm{H}_{20} \mathrm{O}_{3}$ calcd $\mathrm{m} / \mathrm{z} 296.1412$, found $\mathrm{m} / \mathrm{z} 296.1422$.

2-Benzyl-5-benzyloxytetrahydropyran-3-one 5a (minor): insufficient material for complete characterization; $\mathrm{R}_{f} 0.32$ (30\% EtOAc/hexanes); $\mathrm{R}_{t} 16.5 \mathrm{~min}$; IR $\left(\mathrm{CH}_{2} \mathrm{Cl}_{2}\right.$ cast) $1723 \mathrm{~cm}^{-1}$; partial ${ }^{13} \mathrm{C}$ NMR (125 MHz, $\left.\mathrm{CDCl}_{3}\right) \square 207.2,84.4,74.4,70.5,68.2,43.5,35.9$.

Decomposition of 4-Allyloxy-5-benzyloxy-1-diazopentan-2-one $3 \mathrm{~b}$ with $\mathbf{R h}_{\mathbf{2}}(\mathbf{t p a})_{\mathbf{4}}$. The diazoketone $(200 \mathrm{mg}, 0.73 \mathrm{mmol})$ was subjected to the general procedure $\left[\mathrm{Rh}_{2}(\mathrm{tpa})_{4}(17 \mathrm{mg}, 0.013\right.$ $\mathrm{mmol}), \mathrm{CH}_{2} \mathrm{Cl}_{2}(70 \mathrm{~mL}, 0.01 \mathrm{M})$ ] followed by flash chromatography (silica gel, $2 \mathrm{~cm} \times 21 \mathrm{~cm}$ column, 80

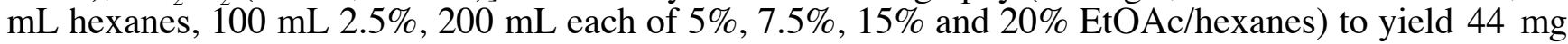
$(25 \%)$ of $\mathbf{4 b}$ (less polar diast.), $47 \mathrm{mg}(26 \%)$ of $\mathbf{4 b}$ (more polar diast.) and $22 \mathrm{mg}$ (12\%) of $7 \mathbf{b}$.

Decomposition of 4-Allyloxy-5-benzyloxy-1-diazopentan-2-one $3 \mathrm{~b}$ with $\mathrm{Cu}(\mathrm{tfacac})_{2}$. The diazoketone $(146 \mathrm{mg}, 0.53 \mathrm{mmol})$ was subjected to the general procedure $\left[\mathrm{Cu}(\mathrm{tfacac})_{2}(20 \mathrm{mg}, 0.054\right.$ $\mathrm{mmol}), \mathrm{CH}_{2} \mathrm{Cl}_{2}(50 \mathrm{~mL}, 0.01 \mathrm{M})$ ] followed by flash chromatography (silica gel, $2 \mathrm{~cm} \mathrm{x} 21 \mathrm{~cm}$ column, $200 \mathrm{~mL}$ each of $10 \%, 20 \%$ and $30 \%$, EtOAc/hexanes) to yield $126 \mathrm{mg}(96 \%)$ of $\mathbf{4 b}$ as a mixture of diastereomers.

2-Allyl-5-(benzyloxymethyl)tetrahydrofuran-3-one $4 \mathrm{~b}$ (less polar): $\mathrm{R}_{f} \quad 0.38 \quad(30 \%$ EtOAc/hexanes); $\mathrm{R}_{t} 11.9 \mathrm{~min}$; IR $\left(\mathrm{CH}_{2} \mathrm{Cl}_{2}\right.$ cast) 3030, 2860, $1756 \mathrm{~cm}^{-1} ;{ }^{1} \mathrm{H}$ NMR $\left(400 \mathrm{MHz}, \mathrm{CDCl}_{3}\right) \square$ 7.36-7.26 (m, 5H), 5.82 (dddd, $J=17.1,10.3,6.8,6.8 \mathrm{~Hz}, 1 \mathrm{H}), 5.18-5.10(\mathrm{~m}, 2 \mathrm{H}), 4.59\left(\mathrm{~d}, J_{\mathrm{AB}}=12.3 \mathrm{~Hz}\right.$, $1 \mathrm{H}), 4.58-4.53(\mathrm{~m}, 1 \mathrm{H}), 4.53\left(\mathrm{~d}, J_{\mathrm{AB}}=12.1 \mathrm{~Hz}, 1 \mathrm{H}\right), 4.15(\mathrm{dd}, J=7.2,4.6 \mathrm{~Hz}, 1 \mathrm{H}), 3.72(\mathrm{dd}, J=10.2$, $3.4 \mathrm{~Hz}, 1 \mathrm{H}), 3.55(\mathrm{dd}, J=10.3,3.7 \mathrm{~Hz}, 1 \mathrm{H}), 2.57-2.45(\mathrm{~m}, 3 \mathrm{H}), 2.35-2.28(\mathrm{~m}, 1 \mathrm{H}) ;{ }^{13} \mathrm{C}$ NMR $(100 \mathrm{MHz}$, 
$\left.\mathrm{CDCl}_{3}\right) \square 215.6,138.0,133.3,128.7,128.0,127.7,118.4,79.6,74.7,73.7,73.0,38.9,36.2 ;$ HRMS (EI) for $\mathrm{C}_{15} \mathrm{H}_{18} \mathrm{O}_{3}$ calcd $\mathrm{m} / \mathrm{z} 246.1256$, found $\mathrm{m} / \mathrm{z} 246.1258$.

2-Allyl-5-(benzyloxymethyl)tetrahydrofuran-3-one $4 \mathbf{b}$ (more polar): $\mathrm{R}_{f} \quad 0.34 \quad(30 \%$ EtOAc/hexanes); $\mathrm{R}_{t} 11.9 \mathrm{~min}$; IR ( $\mathrm{CH}_{2} \mathrm{Cl}_{2}$ cast) $3030,2863,1758 \mathrm{~cm}^{-1} ;{ }^{1} \mathrm{H}$ NMR $\left(400 \mathrm{MHz}, \mathrm{CDCl}_{3}\right) \square$ 7.38-7.27 (m, 5H), $5.84(\mathrm{dddd}, J=17.1,10.1,6.8,6.8 \mathrm{~Hz}, 1 \mathrm{H}), 5.18-5.08(\mathrm{~m}, 2 \mathrm{H}), 4.64\left(\mathrm{~d}, J_{\mathrm{AB}}=12.2 \mathrm{~Hz}\right.$, $1 \mathrm{H}), 4.61\left(\mathrm{~d}, J_{\mathrm{AB}}=12.2 \mathrm{~Hz}, 1 \mathrm{H}\right), 4.41-4.35(\mathrm{~m}, 1 \mathrm{H}), 3.90(\mathrm{dd}, J=6.8,4.5 \mathrm{~Hz}, 1 \mathrm{H}), 3.72-3.61(\mathrm{~m}, 2 \mathrm{H})$, $2.59-2.51(\mathrm{~m}, 1 \mathrm{H}), 2.51(\mathrm{dd}, J=18.1,6.3 \mathrm{~Hz}, 1 \mathrm{H}), 2.38(\mathrm{dd}, J=18.1,9.9 \mathrm{~Hz}, 1 \mathrm{H}), 2.41-2.33(\mathrm{~m}, 1 \mathrm{H})$; ${ }^{13} \mathrm{C}$ NMR $\left(100 \mathrm{MHz}, \mathrm{CDCl}_{3}\right) \square 215.0,138.3,133.3,128.6,127.9,127.9,118.3,81.1,75.2,73.8,71.6$, 39.7, 35.5; HRMS (EI) for $\mathrm{C}_{15} \mathrm{H}_{18} \mathrm{O}_{3}$ calcd $\mathrm{m} / \mathrm{z} 246.1256$, found $\mathrm{m} / \mathrm{z} 246.1257$.

Cyclopropane 7b. $\mathrm{R}_{f} 0.35$ (50\% EtOAc/hexanes); $\mathrm{R}_{t} 12.2 \mathrm{~min}$; IR $\left(\mathrm{CH}_{2} \mathrm{Cl}_{2}\right.$ cast $) 3030,2862,1675$ $\mathrm{cm}^{-1}$; ${ }^{1} \mathrm{H}$ NMR $\left(400 \mathrm{MHz}, \mathrm{CDCl}_{3}\right) \square 7.36-7.26(\mathrm{~m}, 5 \mathrm{H}), 4.56(\mathrm{~s}, 2 \mathrm{H}), 4.45(\mathrm{dd}, J=13.2,2.7 \mathrm{~Hz}, 1 \mathrm{H})$, $3.85(\mathrm{~d}, J=13.3 \mathrm{~Hz}, 1 \mathrm{H}), 3.47(\mathrm{dd}, J=10.0,5.7 \mathrm{~Hz}, 1 \mathrm{H}), 3.42(\mathrm{dd}, J=10.2,4.7 \mathrm{~Hz}, 1 \mathrm{H}), 3.32-3.29(\mathrm{~m}$, $1 \mathrm{H}), 2.70$ (dd, $J=13.6,11.5 \mathrm{~Hz}, 1 \mathrm{H}), 2.37$ (app d, $J=13.6 \mathrm{~Hz}, 1 \mathrm{H}), 2.00$ (dddd, $J=8.6,8.6,6.1,1.7 \mathrm{~Hz}$, $1 \mathrm{H}), 1.62(\mathrm{dd}, J=12.4,6.1 \mathrm{~Hz}, 1 \mathrm{H}), 1.44$ (ddd, $J=15.5,8.9,2.5 \mathrm{~Hz}, 1 \mathrm{H}), 1.19-1.13(\mathrm{~m}, 1 \mathrm{H}) ;{ }^{13} \mathrm{C}$ NMR $\left(100 \mathrm{MHz}, \mathrm{CDCl}_{3}\right) \square$ 209.2, 138.1, 128.6, 127.9, 127.8, 76.1, 73.6, 73.0, 69.1, 46.2, 30.8, 20.3, 11.7; HRMS (EI) for $\mathrm{C}_{15} \mathrm{H}_{18} \mathrm{O}_{3}$ calcd m/z 246.1256, found m/z.246.1255.

Decomposition of 5-Allyloxy-4-benzyloxy-1-diazopentan-2-one $3 \mathrm{c}$ with $\mathrm{Cu}(\mathrm{tfacac})_{2}$. To a mixture of $\mathrm{CH}_{2} \mathrm{Cl}_{2}(55 \mathrm{~mL}, 0.01 \mathrm{M})$ and $\mathrm{Cu}(\mathrm{tfacac})_{2}(19 \mathrm{mg}, 0.055 \mathrm{mmol}, 10 \mathrm{~mol} \%)$ heated to reflux was added via cannula a solution of 5-allyloxy-4-benzyloxy-1-diazopentan-2-one (155 $\mathrm{mg}, 0.55 \mathrm{mmol})$ in $\mathrm{CH}_{2} \mathrm{Cl}_{2}(5.5 \mathrm{~mL})$. When the addition was complete $(5 \mathrm{~min})$ the reaction was monitored by TLC and, upon consumption of the diazoketone $\left(2 \mathrm{~h}\right.$ ), the reaction was cooled to r.t. and $50 \mathrm{~mL}$ of $0.5 \mathrm{M} \mathrm{K}_{2} \mathrm{CO}_{3}$ was added. The aqueous phase was extracted with $\mathrm{CH}_{2} \mathrm{Cl}_{2}(2 \times 50 \mathrm{~mL})$, the combined organic layers were dried $\left(\mathrm{MgSO}_{4}\right)$, filtered, concentrated and purified by flash chromatography (silica gel, $2 \mathrm{~cm} \mathrm{x} 21 \mathrm{~cm}$ column, $200 \mathrm{~mL}$ each of 5\%, 10\% and 15\%, EtOAc/Hexanes) to yield $16 \mathrm{mg}$ (12\%) of $\mathbf{6 c}, 53 \mathrm{mg}(40 \%)$ of $\mathbf{5 c}$ (major diast.), $12 \mathrm{mg}(9 \%)$ of $\mathbf{4 c}$ (less polar diast.), $9 \mathrm{mg}(7 \%)$ of $\mathbf{4 c}$ (more polar diast.), and $37 \mathrm{mg}$ $(28 \%)$ of $\mathbf{5 c}$ (minor diast.).

Enol ether 6c: $\mathrm{R}_{f} 0.46(30 \% \mathrm{EtOAc} /$ hexanes $) ; \mathrm{IR}\left(\mathrm{CH}_{2} \mathrm{Cl}_{2}\right.$ cast $) 3435,3031,2922,2860 \mathrm{~cm}^{-1}$; ${ }^{1} \mathrm{H}$ NMR (500 MHz, $\left.\mathrm{CDCl}_{3}\right) \square 7.37-7.31(\mathrm{~m}, 5 \mathrm{H}), 5.97-5.96(\mathrm{~m}, 1 \mathrm{H}), 5.92$ (dddd, $J=17.2,10.4,5.6,5.6 \mathrm{~Hz}$, $1 \mathrm{H}), 5.29$ (dddd, $J=17.2,1.6,1.6,1.6 \mathrm{~Hz}, 1 \mathrm{H}), 5.20$ (dddd, $J=10.4,1.2,1.2,1.2 \mathrm{~Hz}, 1 \mathrm{H}), 4.70(\mathrm{~s}, 2 \mathrm{H})$, 4.69-4.64 (m, 1H), 4.08-4.05 (m, 2H), $3.60(\mathrm{dd}, J=10.4,6.7 \mathrm{~Hz}, 1 \mathrm{H}), 3.50(\mathrm{dd}, J=10.4,4.4 \mathrm{~Hz}, 1 \mathrm{H})$, $2.84(\mathrm{ddd}, J=14.8,10.4,2.1 \mathrm{~Hz}, 1 \mathrm{H}), 2.61$ (ddd, $J=14.8,8.1,2.1 \mathrm{~Hz}, 1 \mathrm{H}) ;{ }^{13} \mathrm{C} \mathrm{NMR}(125 \mathrm{MHz}$, $\left.\mathrm{CDCl}_{3}\right) \square 141.5,136.9,134.9,128.8,128.4,127.9,121.6,117.6,78.1,72.7,72.5,72.4,33.4$; HRMS (EI) for $\mathrm{C}_{15} \mathrm{H}_{18} \mathrm{O}_{3}$ calcd $\mathrm{m} / \mathrm{z} 246.1256$, found $\mathrm{m} / \mathrm{z} 246.1263$.

2-Allyl-5-benzyloxytetrahydropyran-3-one 5c (major): $\mathrm{R}_{f} 0.40$ (30\% EtOAc/hexanes); $\mathrm{R}_{t} 12.3$ min; IR ( $\mathrm{CH}_{2} \mathrm{Cl}_{2}$ cast) 3030, 2864, $1728 \mathrm{~cm}^{-1} ;{ }^{1} \mathrm{H}$ NMR (500 MHz, $\left.\mathrm{CDCl}_{3}\right) \square 7.36-7.29(\mathrm{~m}, 5 \mathrm{H}), 5.82$ (dddd, $J=17.1,10.1,6.9,6.9 \mathrm{~Hz}, 1 \mathrm{H}), 5.15-5.07(\mathrm{~m}, 2 \mathrm{H}), 4.58\left(\mathrm{~d}, J_{A B}=11.7 \mathrm{~Hz}, 1 \mathrm{H}\right), 4.49\left(\mathrm{~d}, J_{A B}=11.8\right.$ $\mathrm{Hz}, 1 \mathrm{H}), 4.24(\mathrm{ddd}, J=11.7,5.6,0.9 \mathrm{~Hz}, 1 \mathrm{H}), 4.06$ (dddd, $J=6.6,5.9,5.9,4.6 \mathrm{~Hz}, 1 \mathrm{H}), 3.86(\mathrm{dd}, J=8.1$, $4.2 \mathrm{~Hz}, 1 \mathrm{H}), 3.66(\mathrm{dd}, J=11.8,5.8 \mathrm{~Hz}, 1 \mathrm{H}), 2.87$ (ddd, $J=15.4,4.6,0.4 \mathrm{~Hz}, 1 \mathrm{H}), 2.62(\mathrm{dd}, J=15.4,6.6$ $\mathrm{Hz}, 1 \mathrm{H}), 2.64-2.58(\mathrm{~m}, 1 \mathrm{H}), 2.39-2.32(\mathrm{~m}, 1 \mathrm{H}) ;{ }^{13} \mathrm{C} \mathrm{NMR}\left(125 \mathrm{MHz}, \mathrm{CDCl}_{3}\right) \square 207.7,137.8,133.9$, 128.7, 128.2, 127.9, 117.8, 81.9, 73.3, 71.1, 69.8, 43.2, 34.4; HRMS (EI) for $\mathrm{C}_{15} \mathrm{H}_{18} \mathrm{O}_{3}$ calcd m/z 246.1256, found $\mathrm{m} / \mathrm{z} 246.1261$.

2-Benzyl-5-(allyloxymethyl)tetrahydrofuran-3-one $4 c$ (less polar): $\quad \mathbf{R}_{f} \quad 0.36 \quad(30 \%$ EtOAc/hexanes); $\mathrm{R}_{t} 12.3 \mathrm{~min}$; IR $\left(\mathrm{CH}_{2} \mathrm{Cl}_{2}\right.$ cast) 3029, 2922, 2852, $1756 \mathrm{~cm}^{-1} ;{ }^{1} \mathrm{H}$ NMR (400 MHz, $\left.\mathrm{CDCl}_{3}\right) \square 7.30-7.20(\mathrm{~m}, 5 \mathrm{H}), 5.85$ (dddd, $\left.J=17.3,10.4,5.5,5.5 \mathrm{~Hz}, 1 \mathrm{H}\right), 5.25-5.15(\mathrm{~m}, 2 \mathrm{H}), 4.36-4.31$ $(\mathrm{m}, 2 \mathrm{H}), 4.03-3.99(\mathrm{~m}, 2 \mathrm{H}), 3.62(\mathrm{dd}, J=10.3,3.4 \mathrm{~Hz}, 1 \mathrm{H}), 3.47(\mathrm{dd}, J=10.3,3.7 \mathrm{~Hz}, 1 \mathrm{H}), 3.02(\mathrm{dd}, J=$ $14.3,4.3 \mathrm{~Hz}, 1 \mathrm{H}), 2.88(\mathrm{dd}, J=14.4,6.6 \mathrm{~Hz}, 1 \mathrm{H}), 2.41(\mathrm{ddd}, J=18.0,4.7,0.8 \mathrm{~Hz}, 1 \mathrm{H}), 2.22(\mathrm{dd}, J=$ 18.0, 8.3 Hz, 1H); ${ }^{13} \mathrm{C}$ NMR (100 MHz, $\left.\mathrm{CDCl}_{3}\right) \square 215.7,137.1,134.4,129.9,128.5,126.8,117.3,80.8$, 74.7, 73.0, 72.6, 39.0, 38.0; HRMS (EI) for $\mathrm{C}_{15} \mathrm{H}_{18} \mathrm{O}_{3}$ calcd m/z 246.1256, found $\mathrm{m} / \mathrm{z} 246.1264$.

2-Benzyl-5-(allyloxymethyl)tetrahydrofuran-3-one $4 c$ (more polar): $R_{f} \quad 0.32 \quad(30 \%$ EtOAc/hexanes); $\mathrm{R}_{t} 11.4 \mathrm{~min}$; IR $\left(\mathrm{CH}_{2} \mathrm{Cl}_{2}\right.$ cast) 3028, 2922, 2852, $1756 \mathrm{~cm}^{-1} ;{ }^{1} \mathrm{H}$ NMR $(400 \mathrm{MHz}$, $\left.\mathrm{CDCl}_{3}\right) \square 7.36-7.19(\mathrm{~m}, 5 \mathrm{H}), 5.89(\mathrm{dddd}, J=17.3,10.4,5.6,5.6 \mathrm{~Hz}, 1 \mathrm{H}), 5.27$ (app dq, $J=17.2,1.6 \mathrm{~Hz}$, 1H), 5.20 (app dq, $J=10.4,1.3 \mathrm{~Hz}, 1 \mathrm{H}) 4.37-4.31(\mathrm{~m}, 1 \mathrm{H}), 4.09$ (dd, $J=7.0,4.0 \mathrm{~Hz}, 1 \mathrm{H}), 4.04$ (app dt, $J$ $=5.6,1.4 \mathrm{~Hz}, 2 \mathrm{H}), 3.55-3.53(\mathrm{~m}, 2 \mathrm{H}), 3.09(\mathrm{dd}, J=14.4,4.0 \mathrm{~Hz}, 1 \mathrm{H}), 2.92(\mathrm{dd}, J=14.5,7.0 \mathrm{~Hz}, 1 \mathrm{H})$, $2.46(\mathrm{dd}, J=17.9,6.2 \mathrm{~Hz}, 1 \mathrm{H}), 2.10(\mathrm{dd}, J=18.0,9.7 \mathrm{~Hz}, 1 \mathrm{H}) ;{ }^{13} \mathrm{C} \mathrm{NMR}\left(100 \mathrm{MHz}, \mathrm{CDCl}_{3}\right) \square 215.0$, 
137.2, 134.6, 129.9, 128.4, 126.8, 117.5, 82.1, 75.1, 72.7, 71.9, 39.8, 37.5; HRMS (EI) for $\mathrm{C}_{15} \mathrm{H}_{18} \mathrm{O}_{3}$ calcd $\mathrm{m} / \mathrm{z} 246.1256$, found $\mathrm{m} / \mathrm{z} 246.1266$.

2-Allyl-5-benzyloxytetrahydropyran-3-one 5c (minor): $\mathbf{R}_{f} 0.28$ (30\% EtOAc/hexanes); $\mathbf{R}_{t} 12.3$ min; IR ( $\mathrm{CH}_{2} \mathrm{Cl}_{2}$ cast) 3065, 2971, 2862, $1727 \mathrm{~cm}^{-1} ;{ }^{1} \mathrm{H}$ NMR (400 MHz, $\left.\mathrm{CDCl}_{3}\right)$ 7.38-7.27 (m, 5H), 5.87 (dddd, $J=17.2,10.1,7.0,7.0 \mathrm{~Hz}, 1 \mathrm{H}), 5.18-5.08(\mathrm{~m}, 2 \mathrm{H}), 4.57$ (s, 2H), 4.16 (ddd, $J=12.6,2.7,2.0$ $\mathrm{Hz}, 1 \mathrm{H}), 4.03-3.99(\mathrm{~m}, 1 \mathrm{H}), 3.87$ (dd, $J=8.2,4.3 \mathrm{~Hz}, 1 \mathrm{H}), 3.78$ (dd, $J=12.6,2.9 \mathrm{~Hz}, 1 \mathrm{H}), 2.79$ (ddd, $J=$ $15.6,4.4,1.9 \mathrm{~Hz}, 1 \mathrm{H}), 2.69(\mathrm{ddd}, J=15.6,5.0,0.6 \mathrm{~Hz}, 1 \mathrm{H}), 2.65-2.56(\mathrm{~m}, 1 \mathrm{H}), 2.51-2.43(\mathrm{~m}, 1 \mathrm{H}) ;{ }^{13} \mathrm{C}$ NMR $\left(100 \mathrm{MHz} \mathrm{CDCl}_{3}\right) \square 207.1,137.9,134.0,128.7,128.1,127.9,117.8,82.9,74.0,70.5,68.0,43.5$, 34.2; HRMS (EI) for $\mathrm{C}_{15} \mathrm{H}_{18} \mathrm{O}_{3}$ calcd $\mathrm{m} / \mathrm{z} 246.1256$, found $\mathrm{m} / \mathrm{z} 246.1253$.

1. Aldrich Chemical Co., Milwaukee, WI: Technical Information Bulletin No. AL-113.

2. Beck, G.; Jendralla, H.; Kesseler, K. Synthesis 1995, 1014-1018.

3. Langer, P.; Eckardt, T. Synlett 2000, 844-846. 


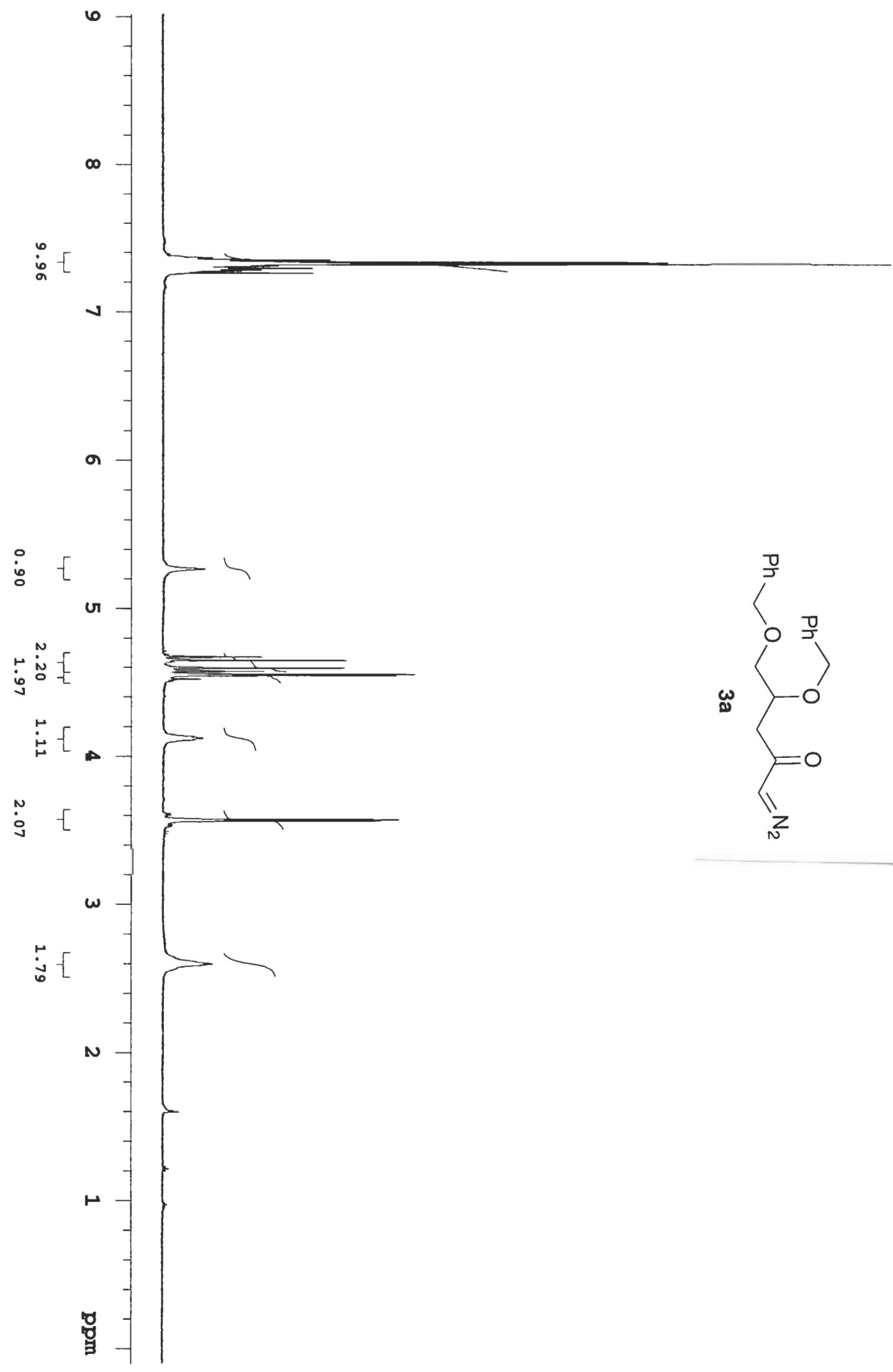




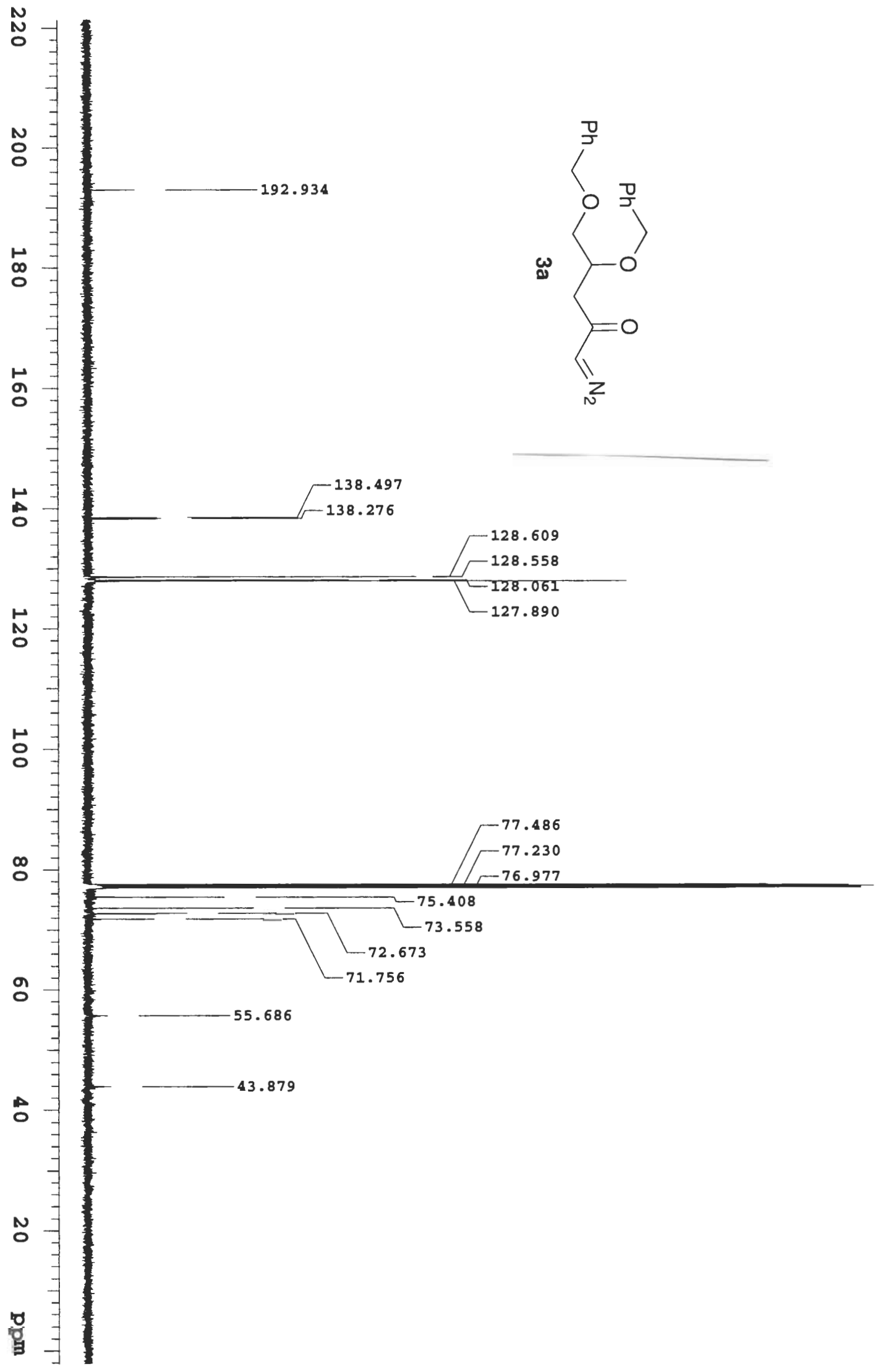




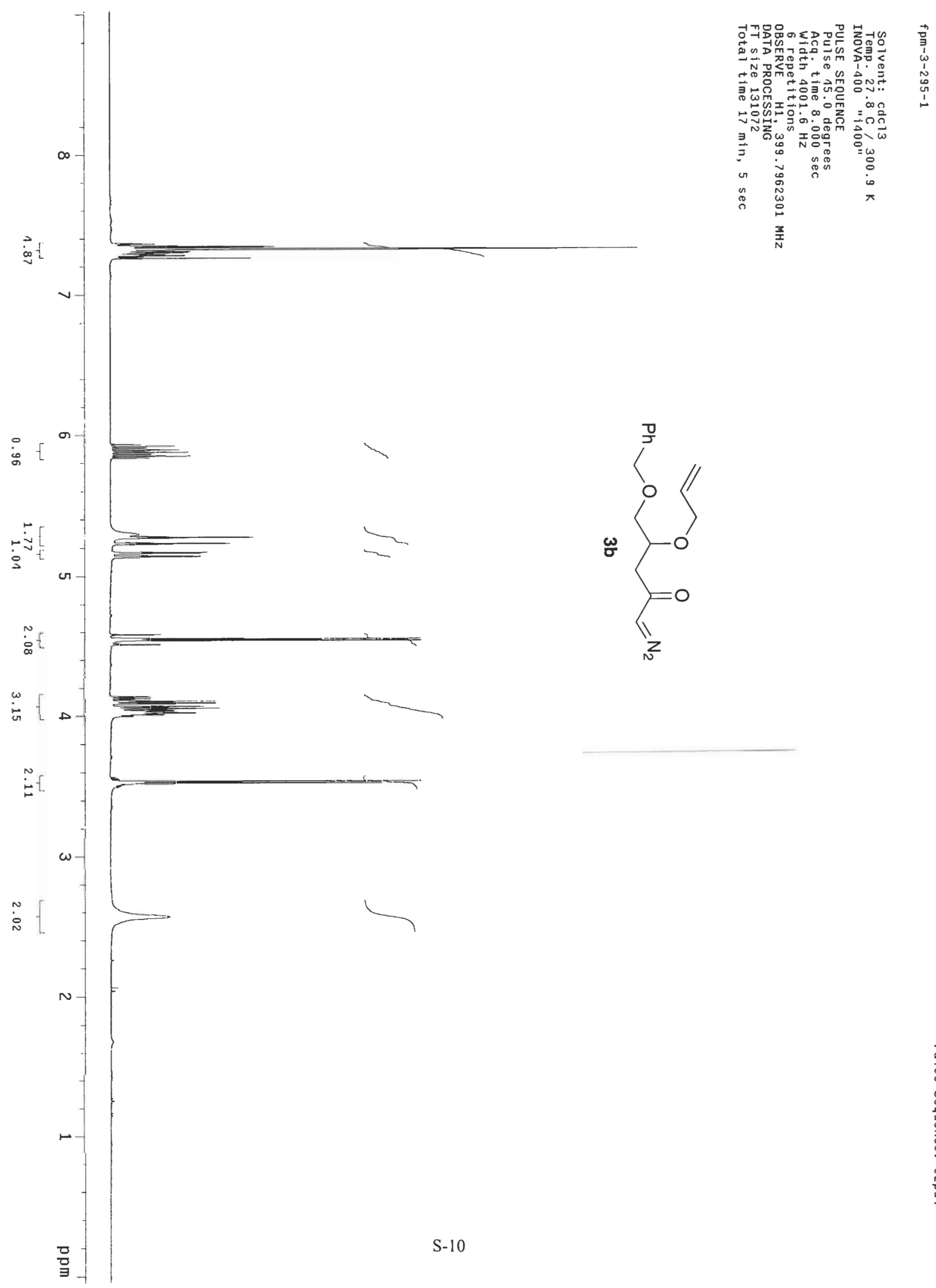




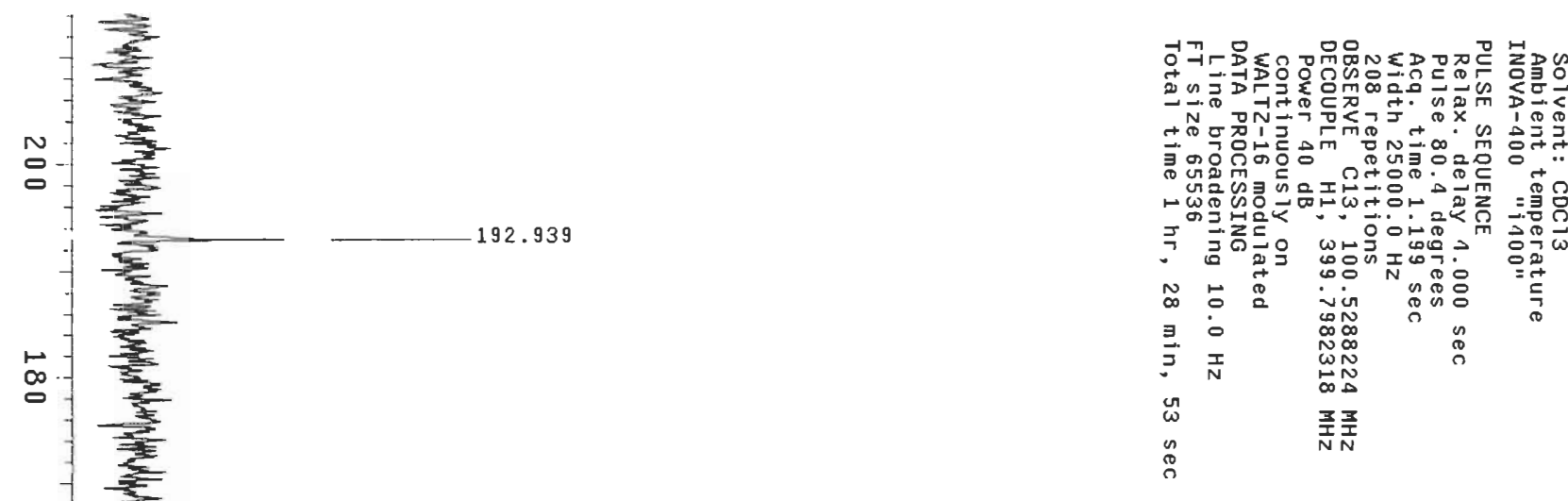

$-128.581$

$-127.867$

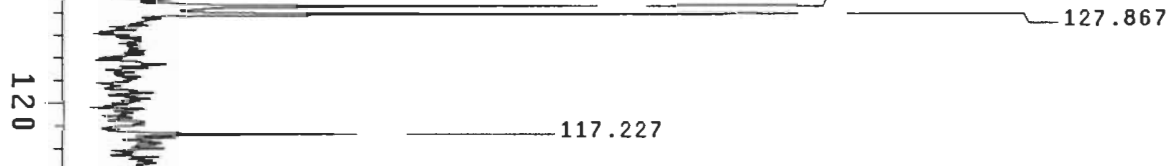

$\stackrel{\infty}{\circ}$

138.257 135.001

$$
\text { 뭉 }
$$

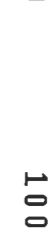

․․

青$$
\text { . }
$$

.
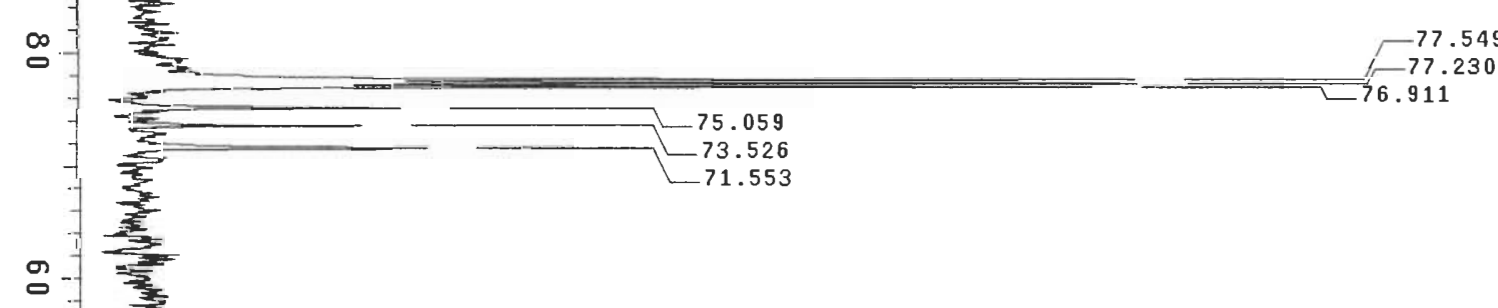

55.661

43.776

b

를 


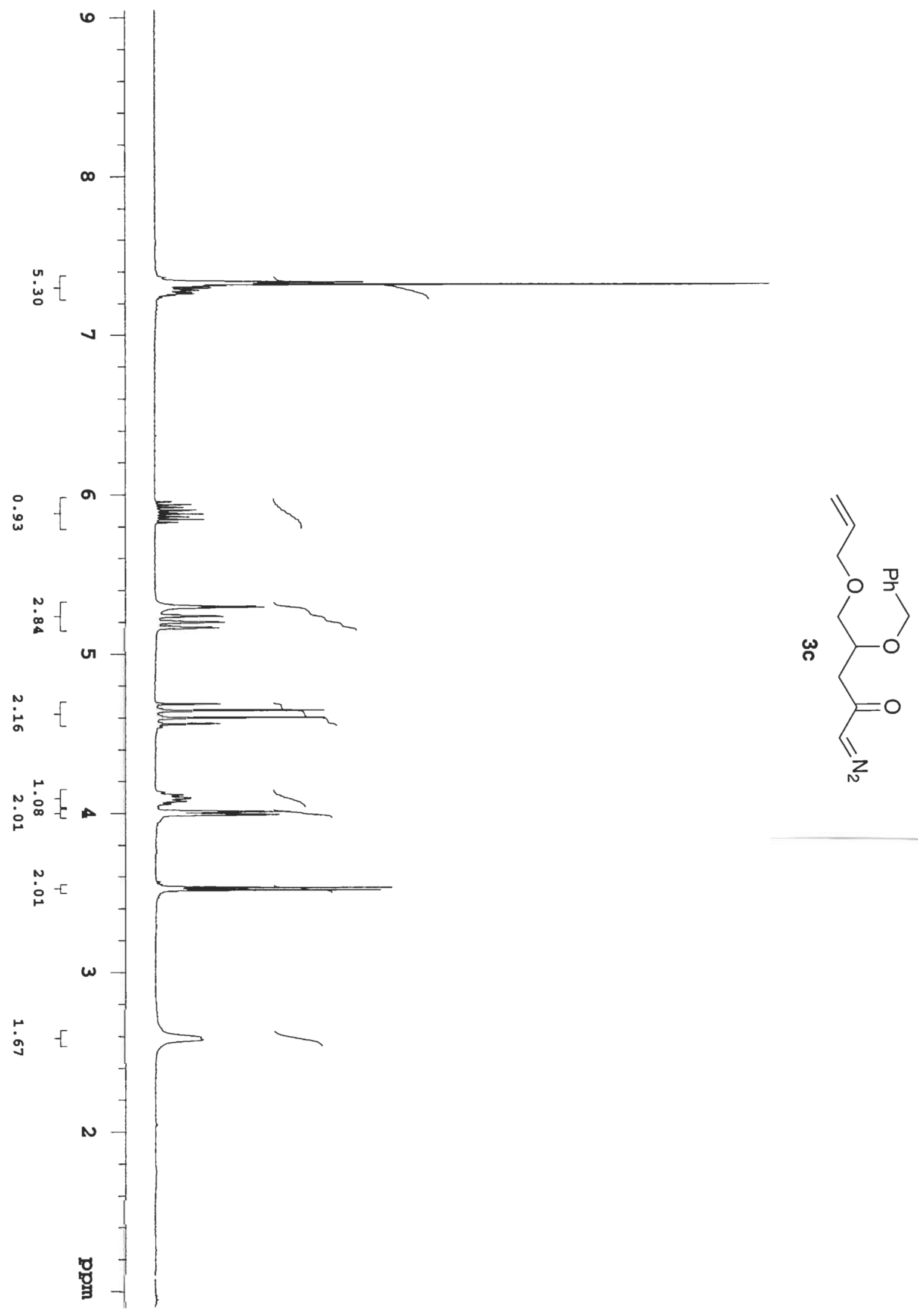

S- 12 


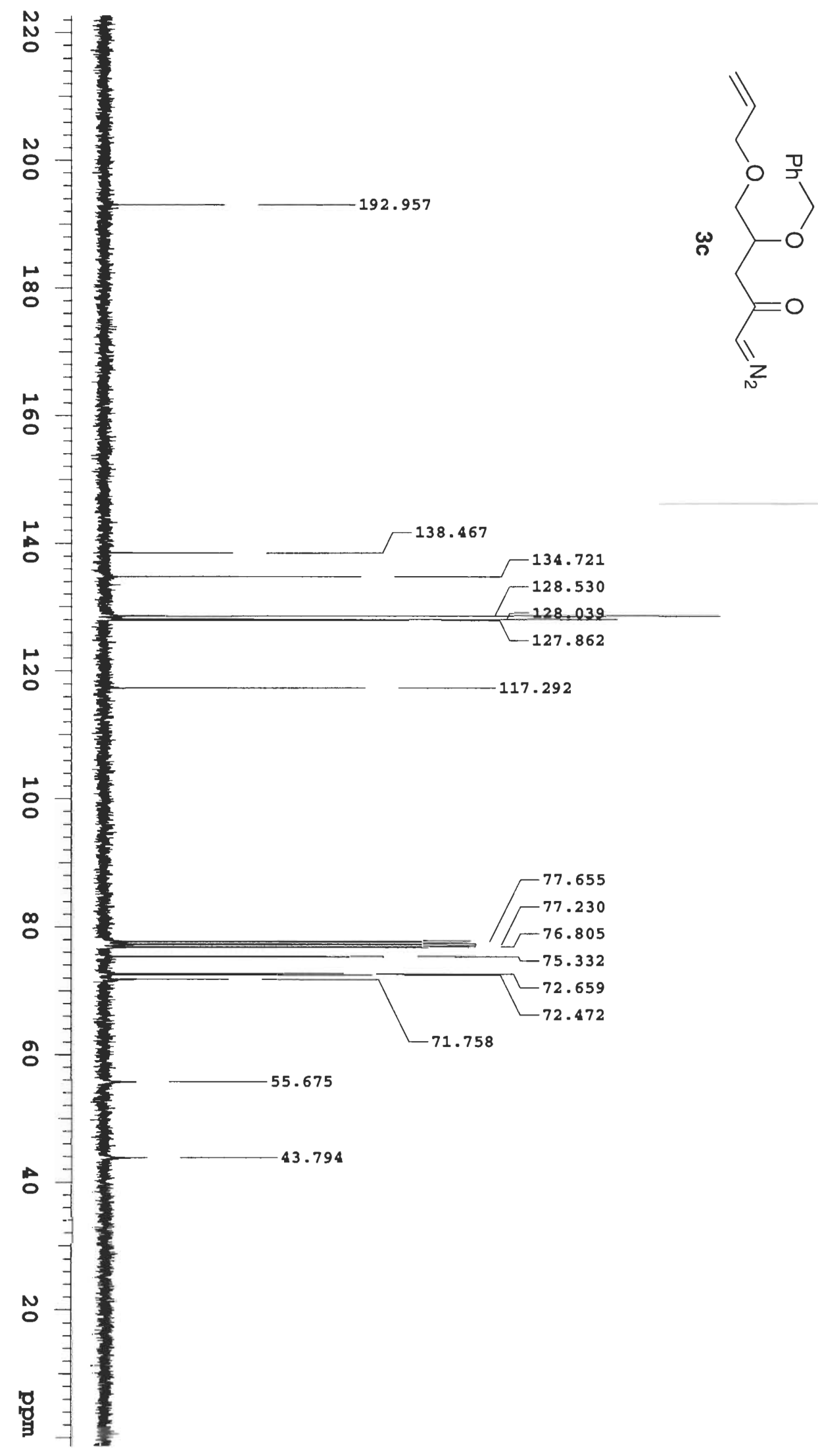




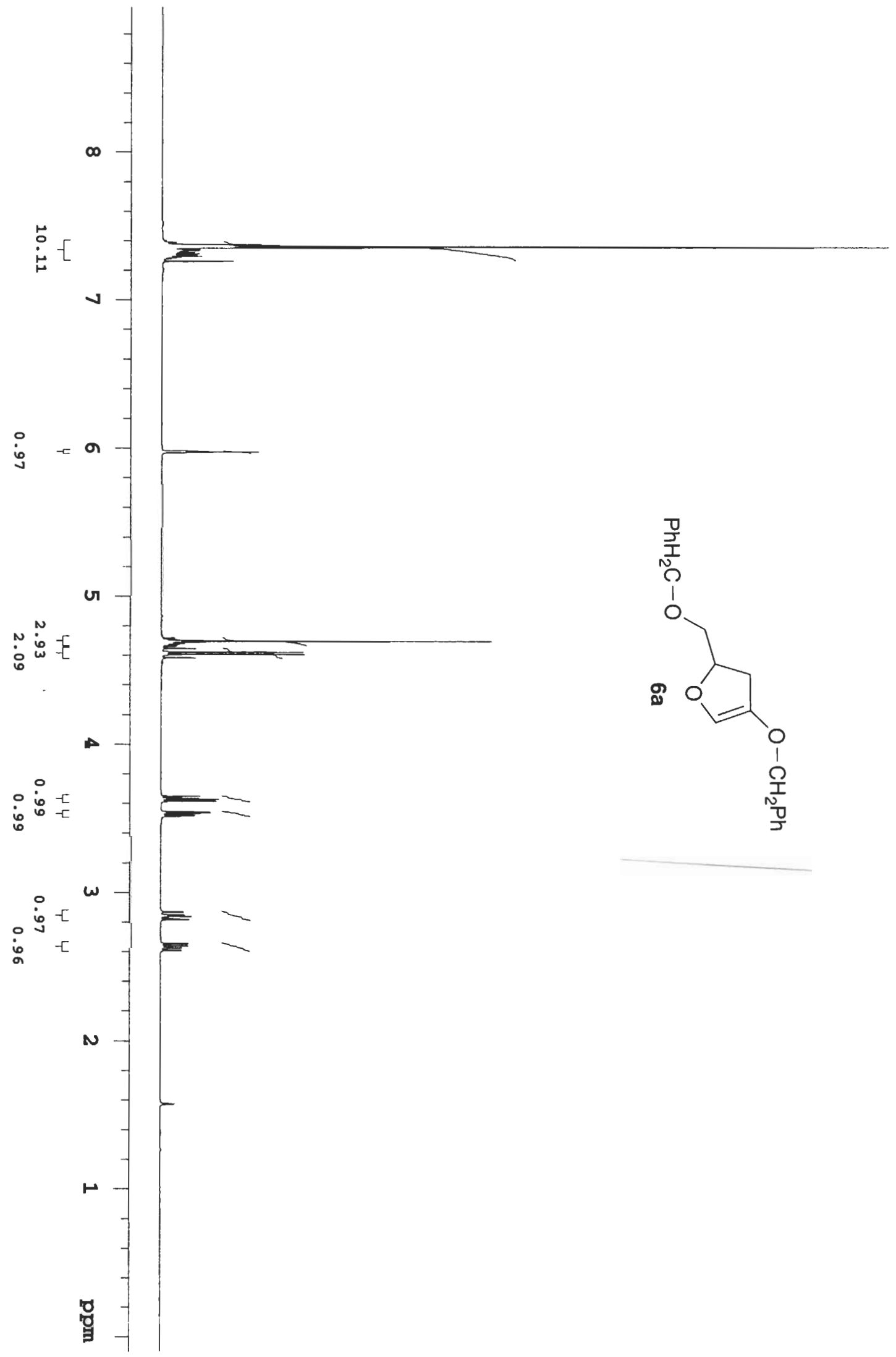




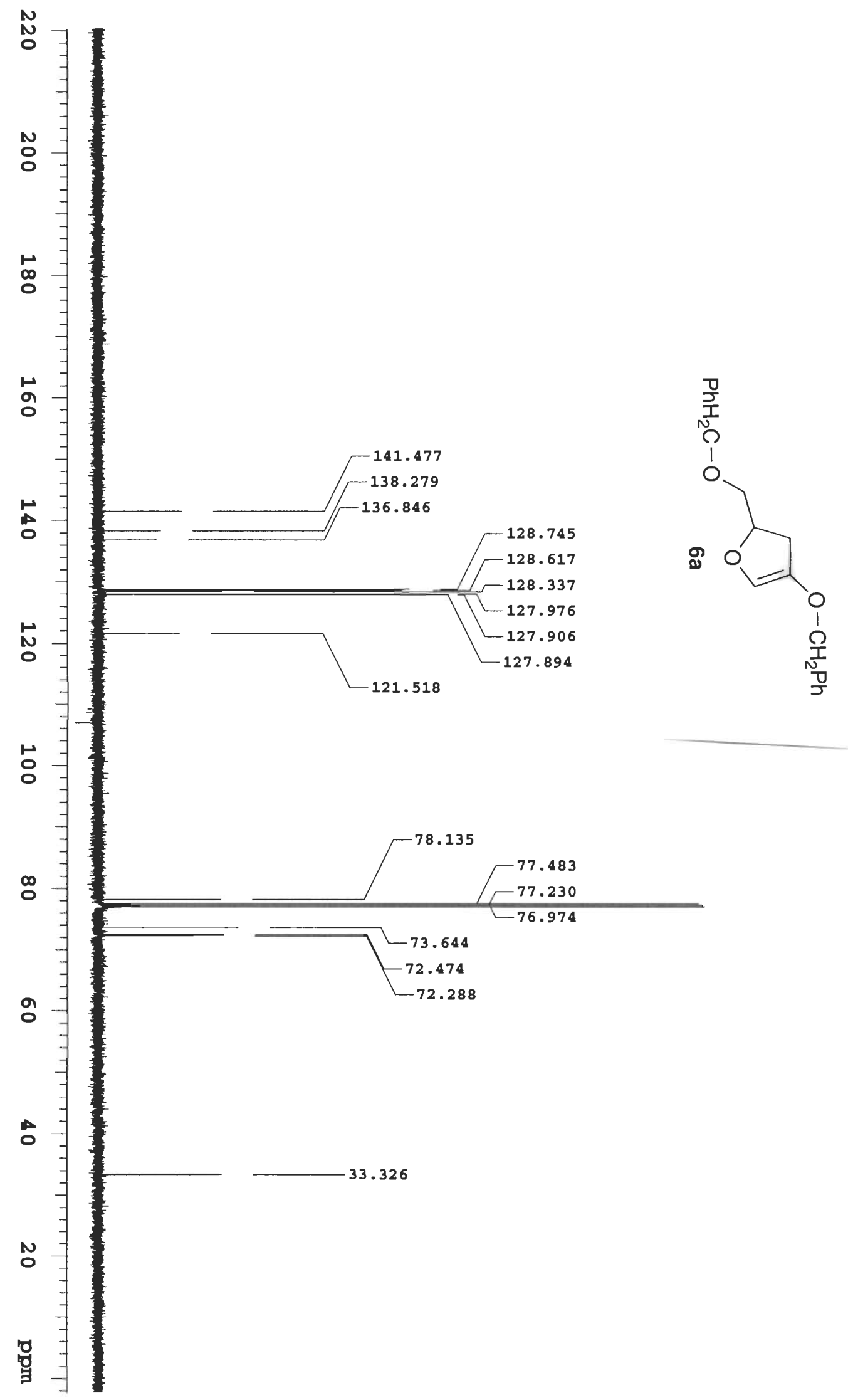



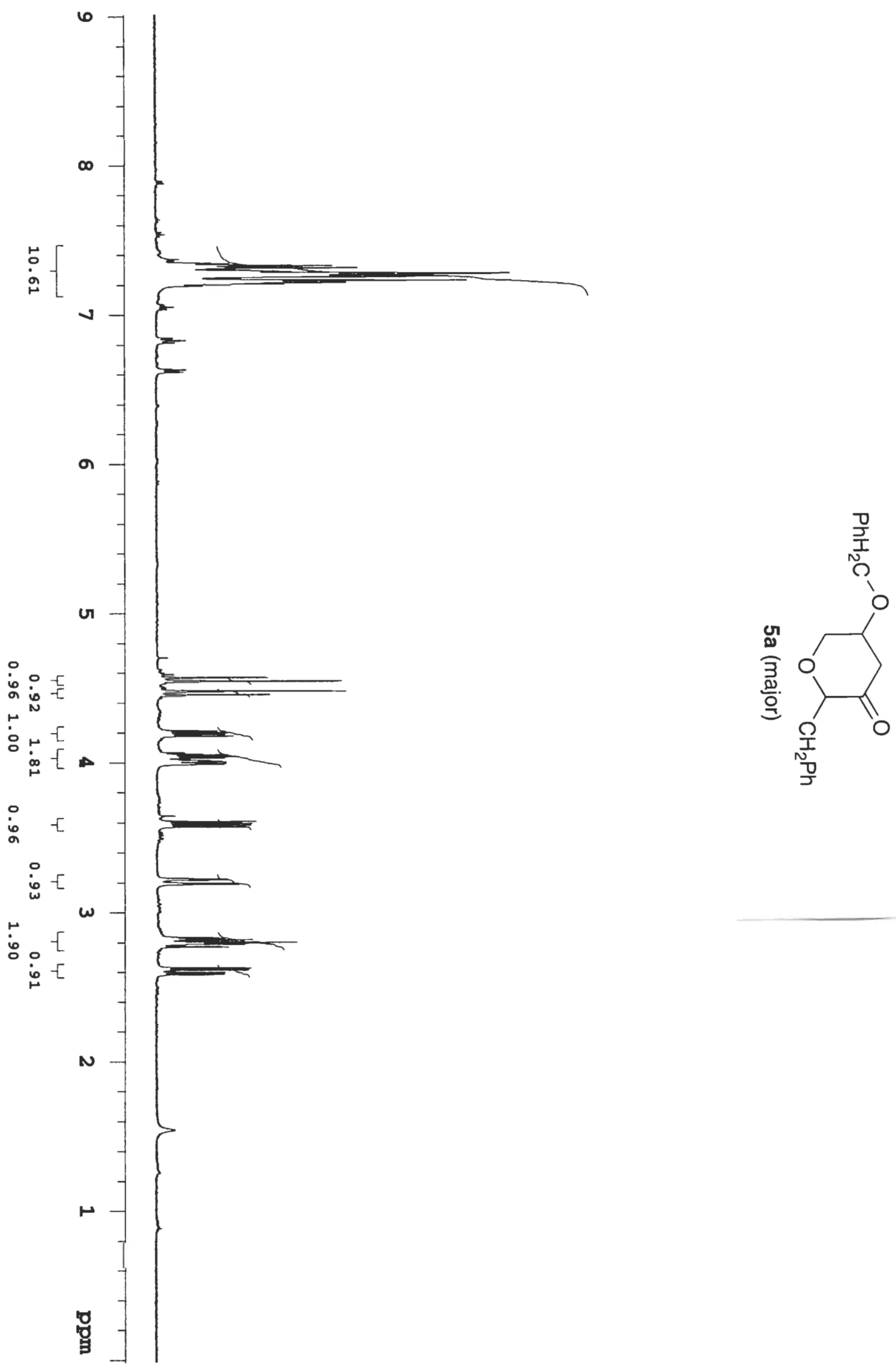


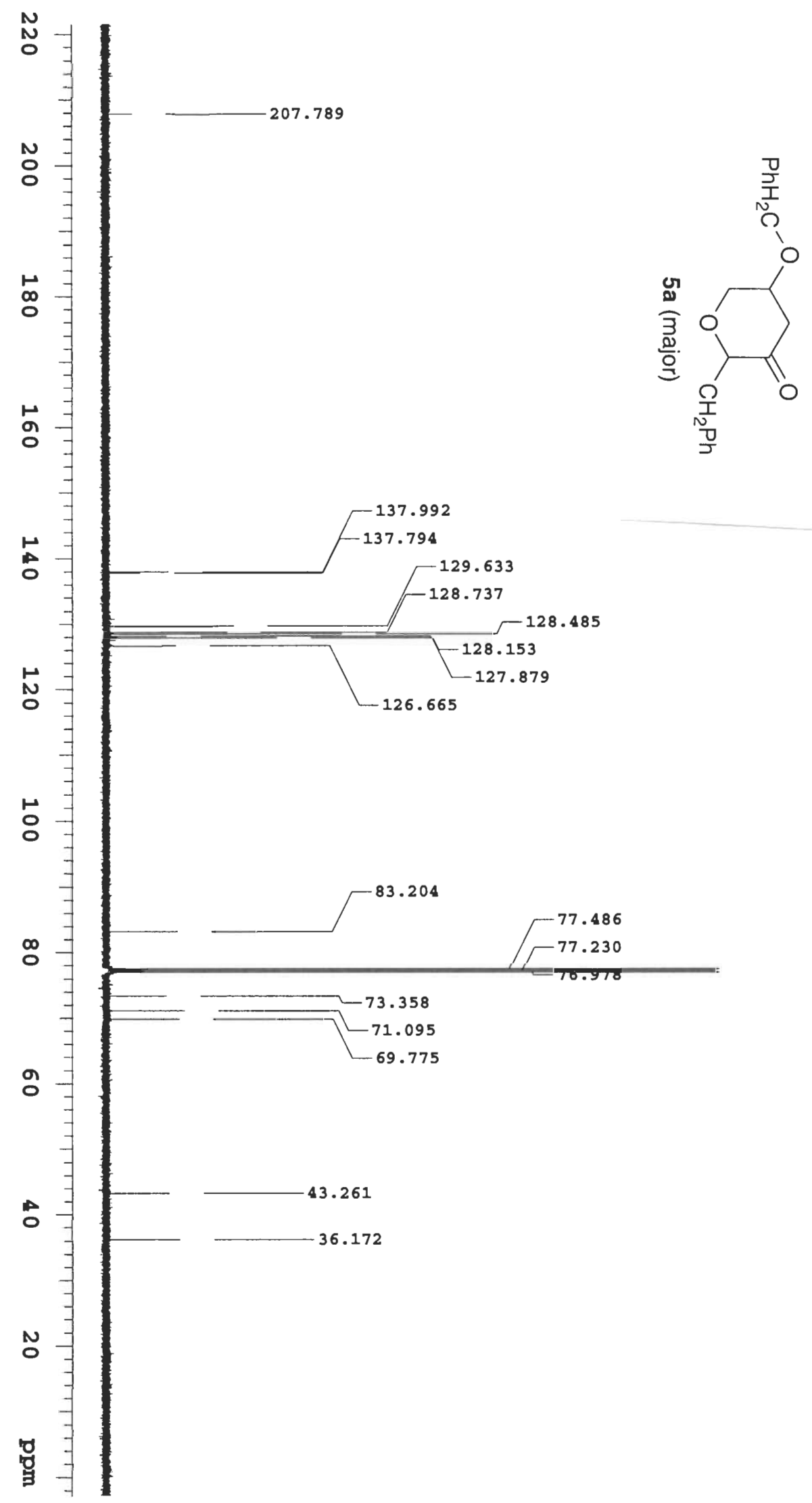




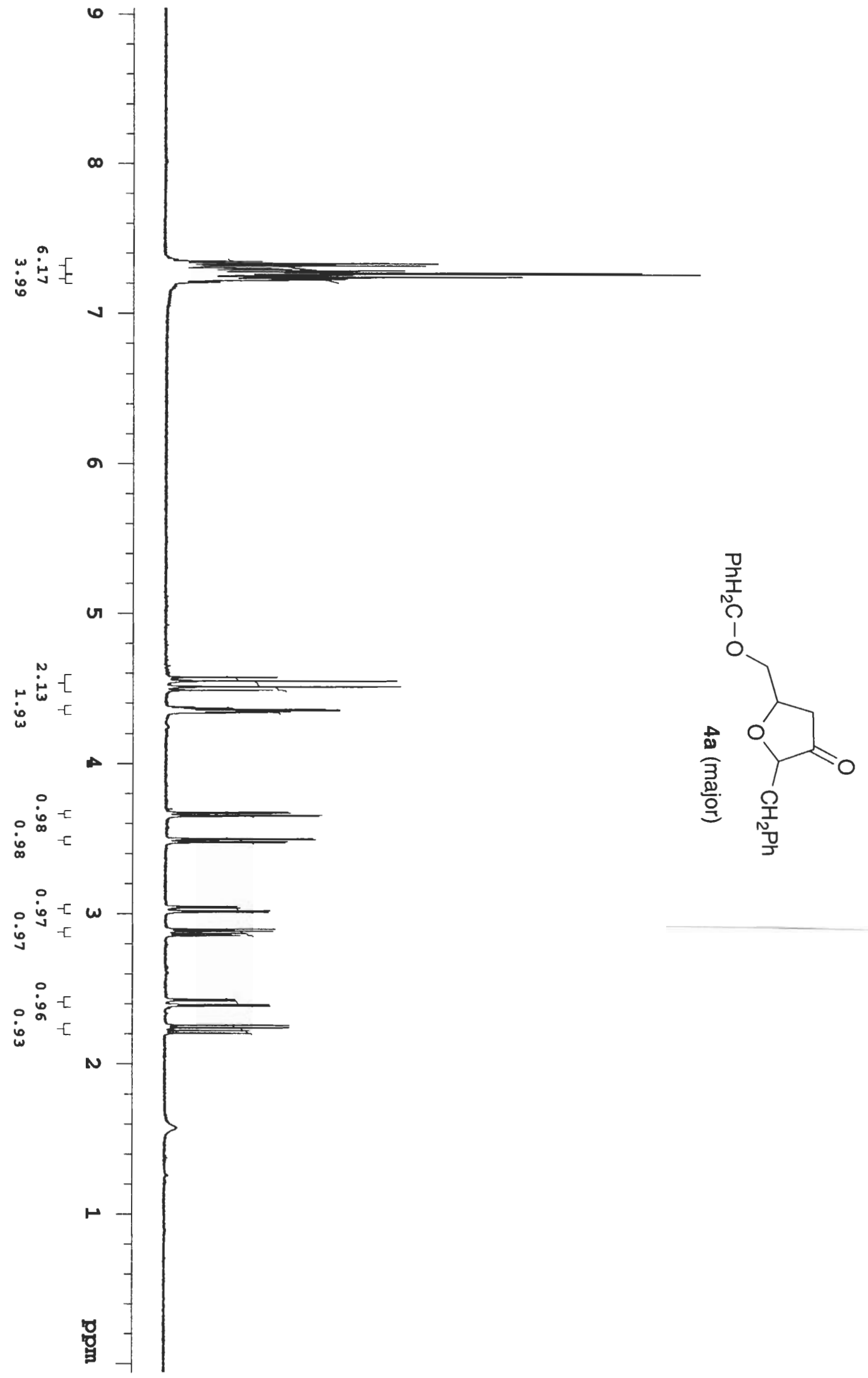




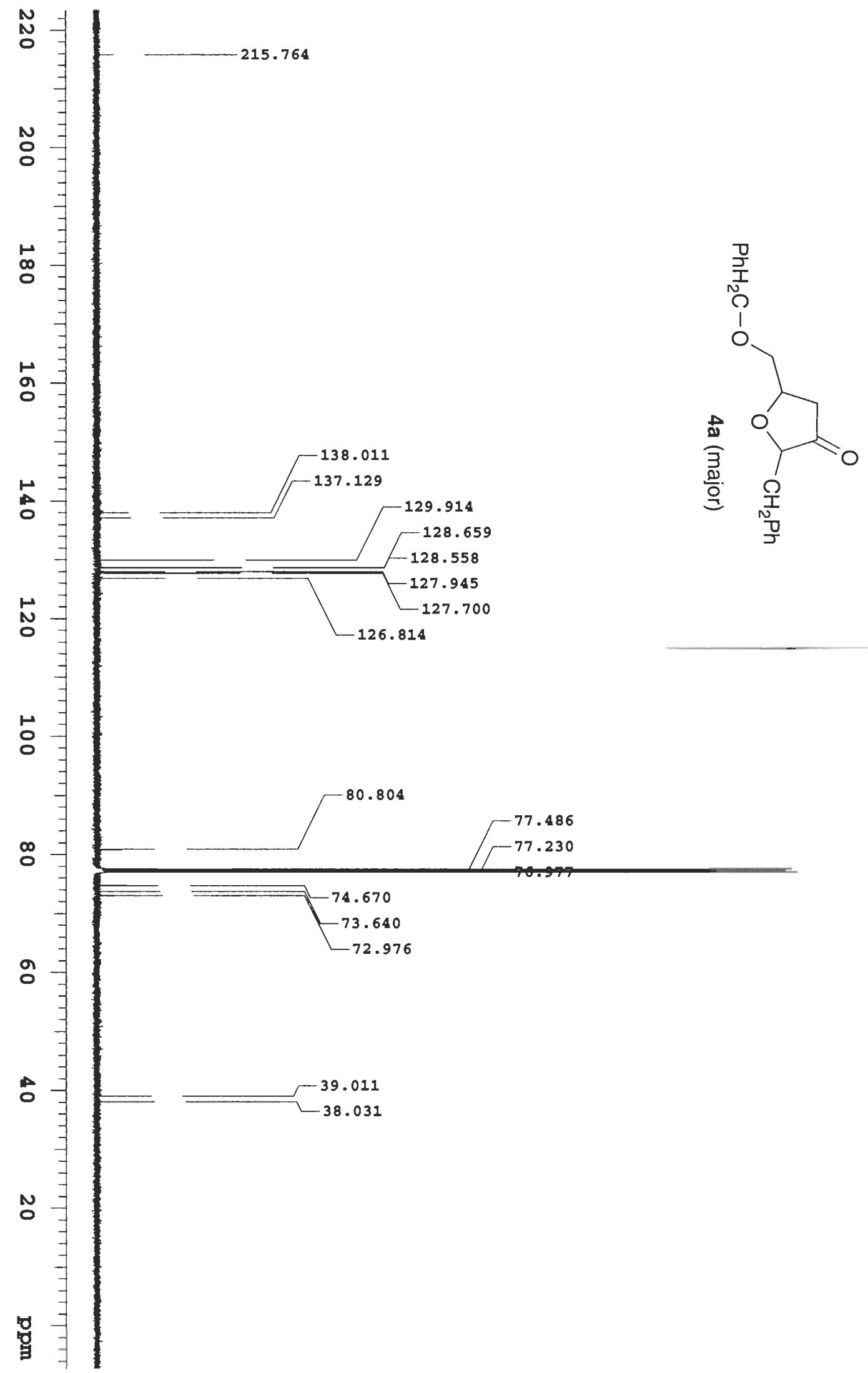




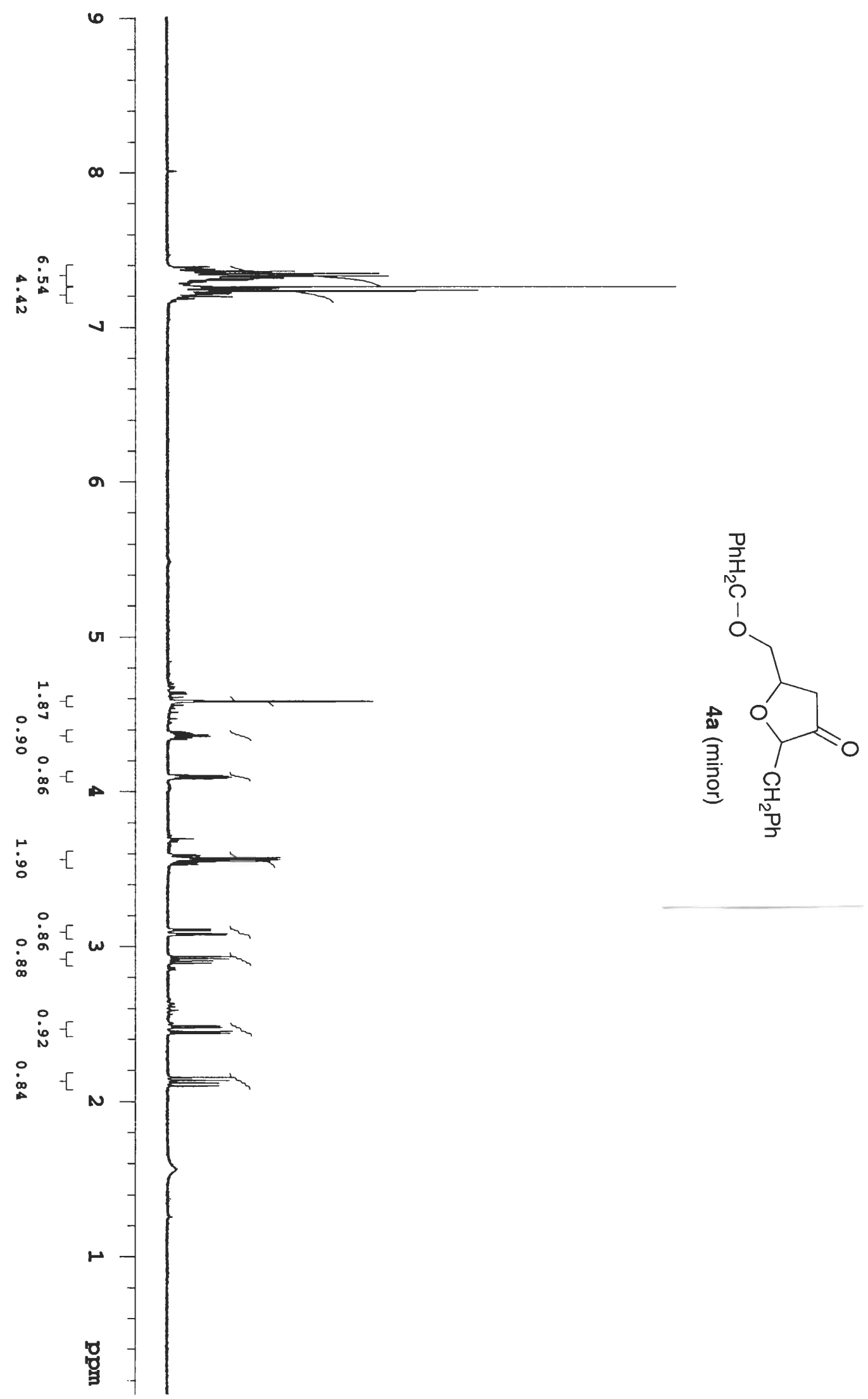




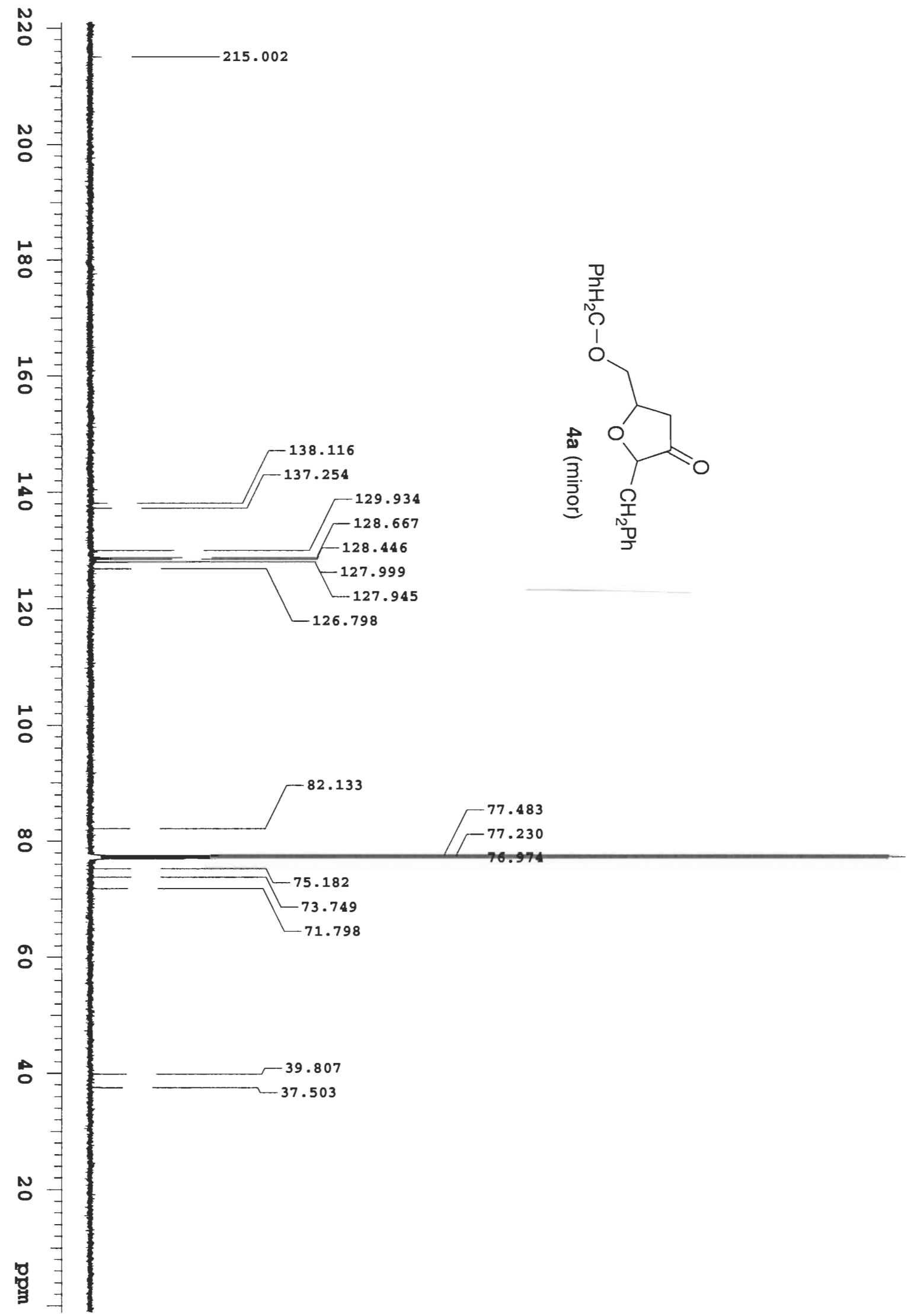



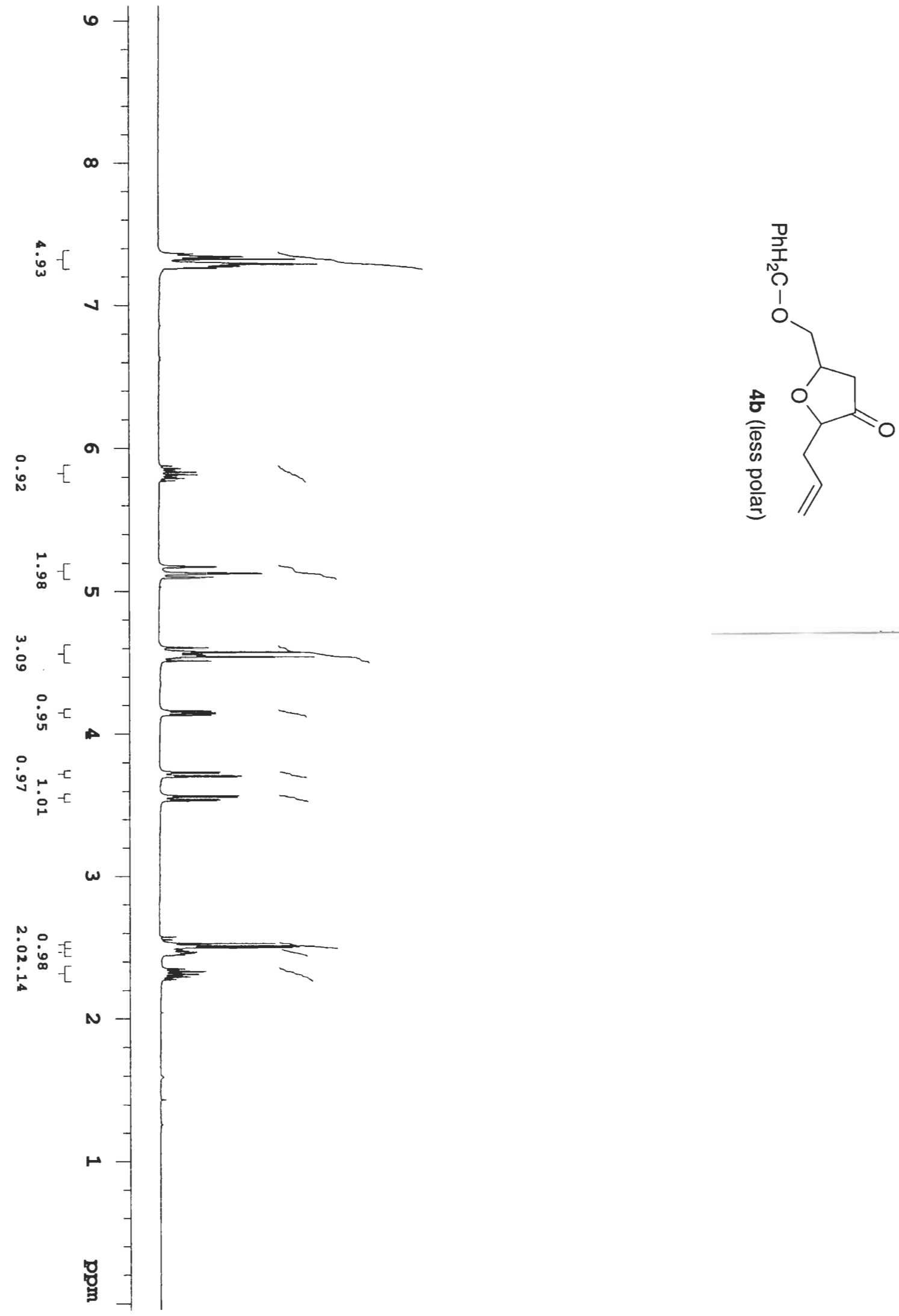


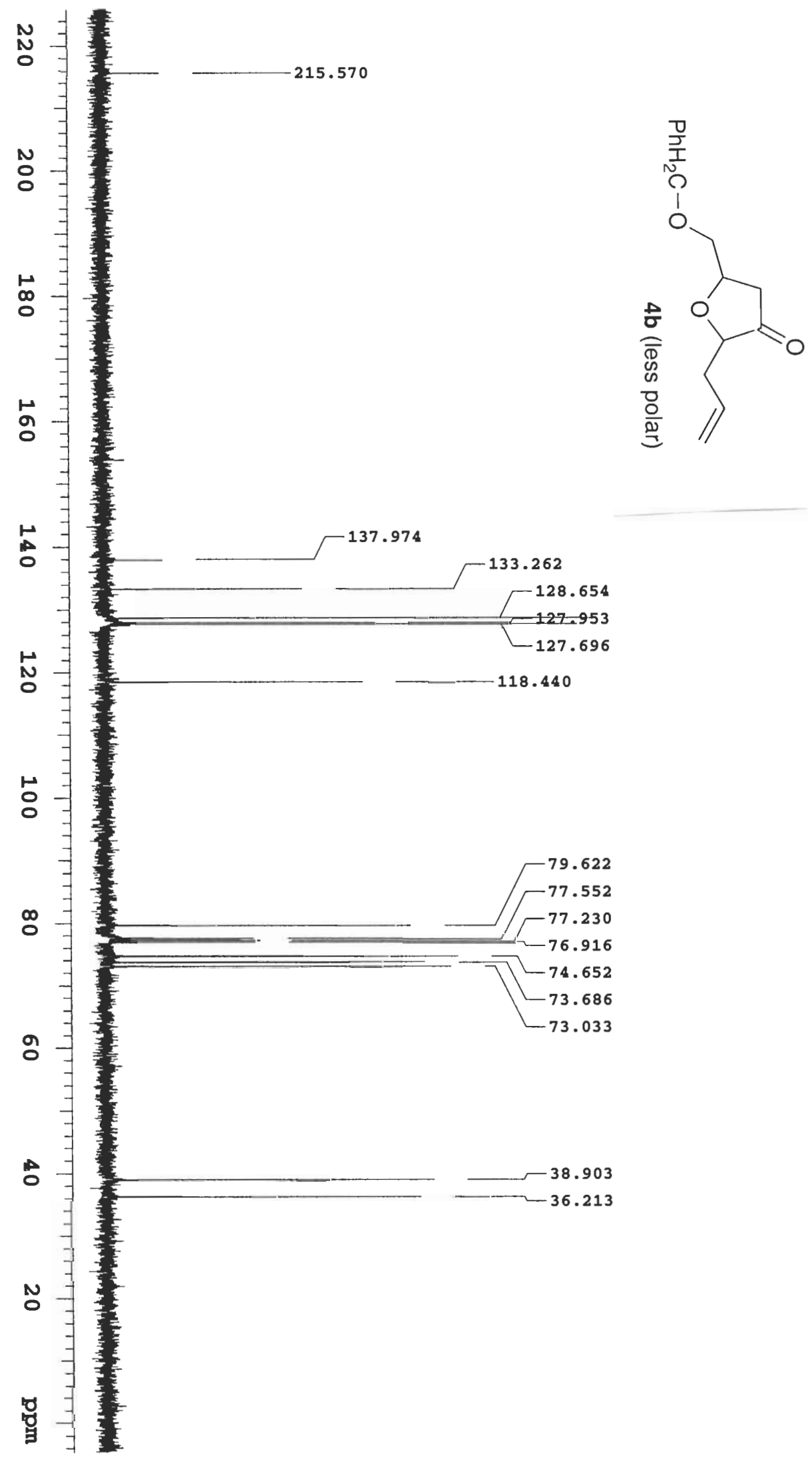



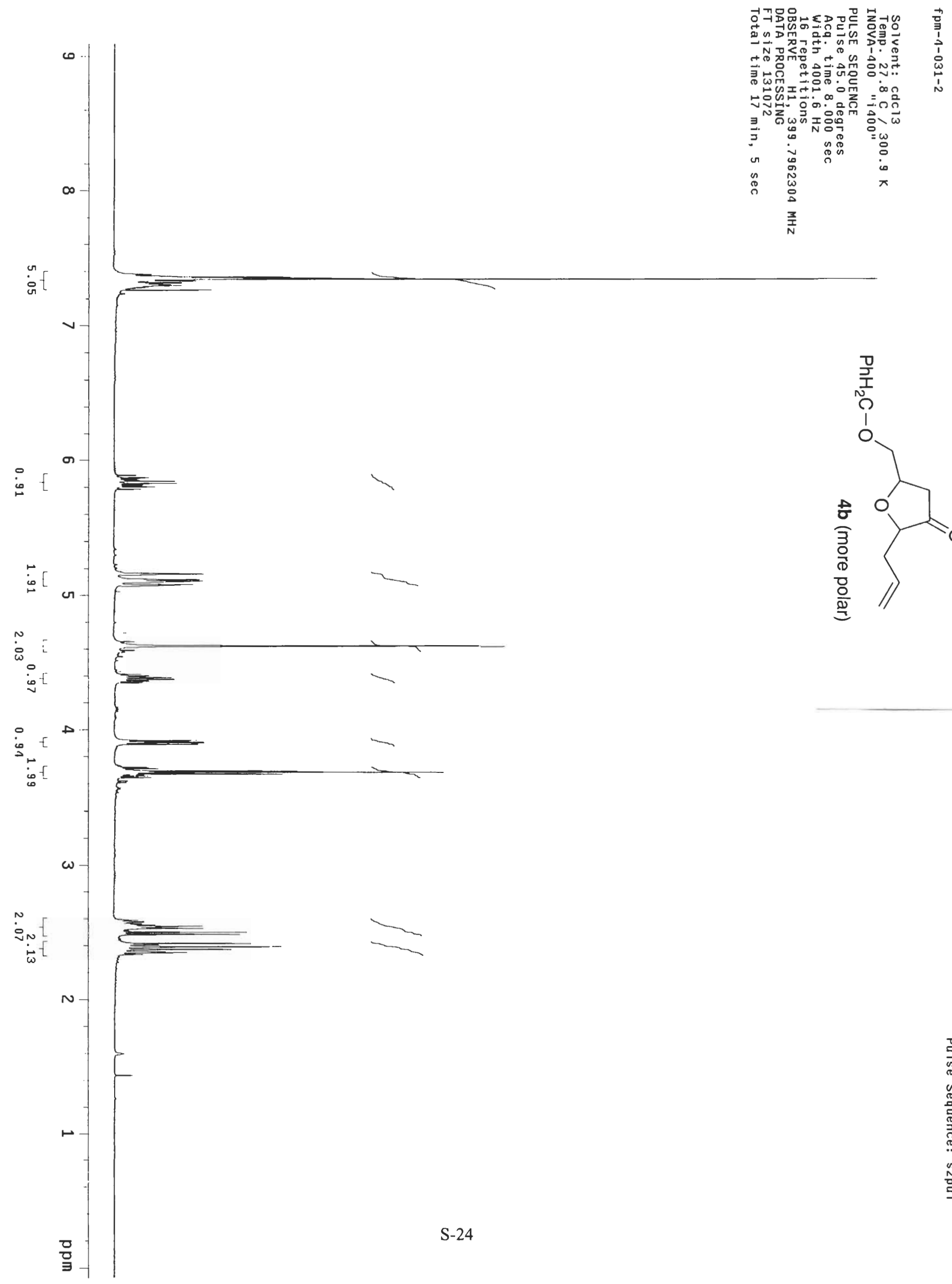


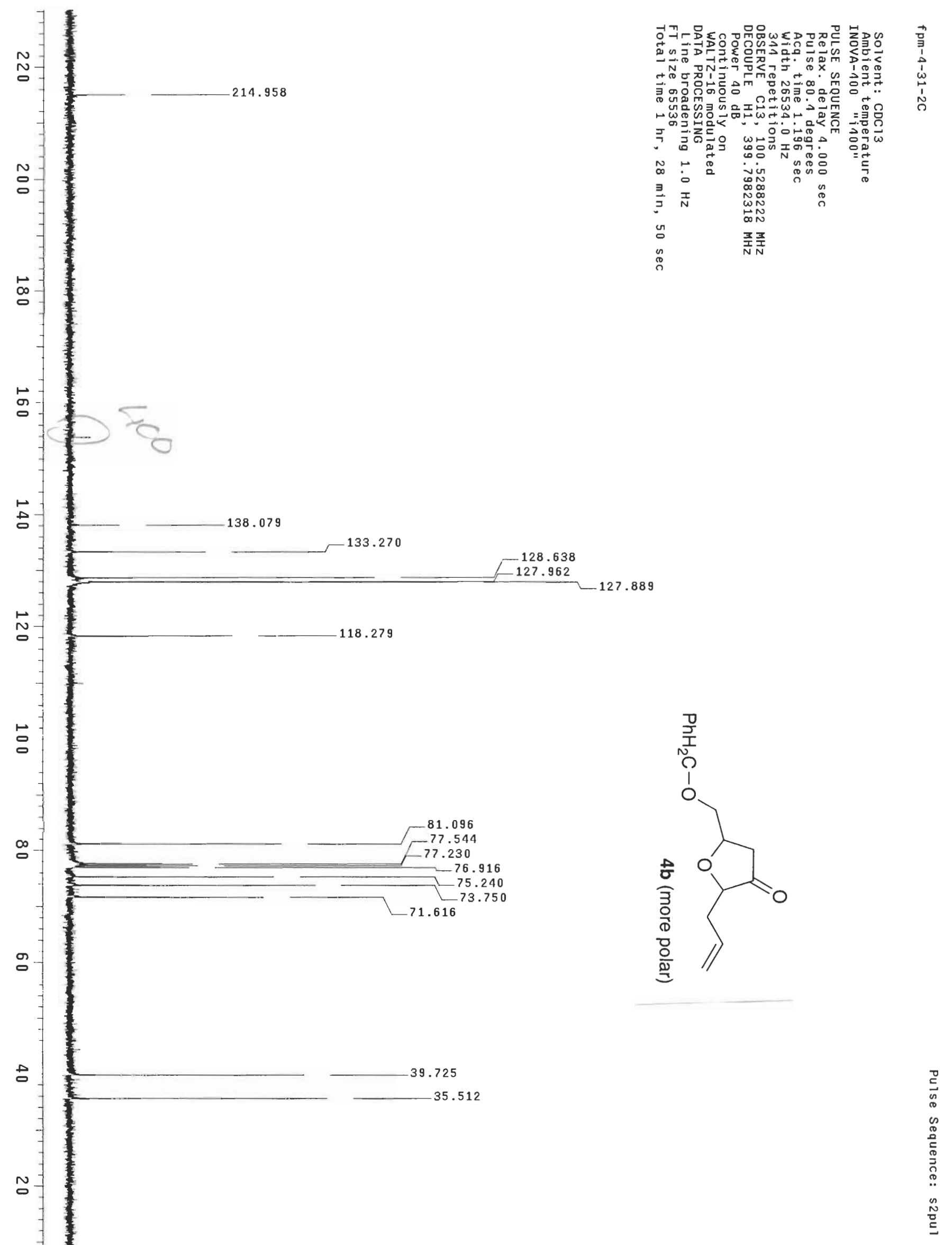

S-25 

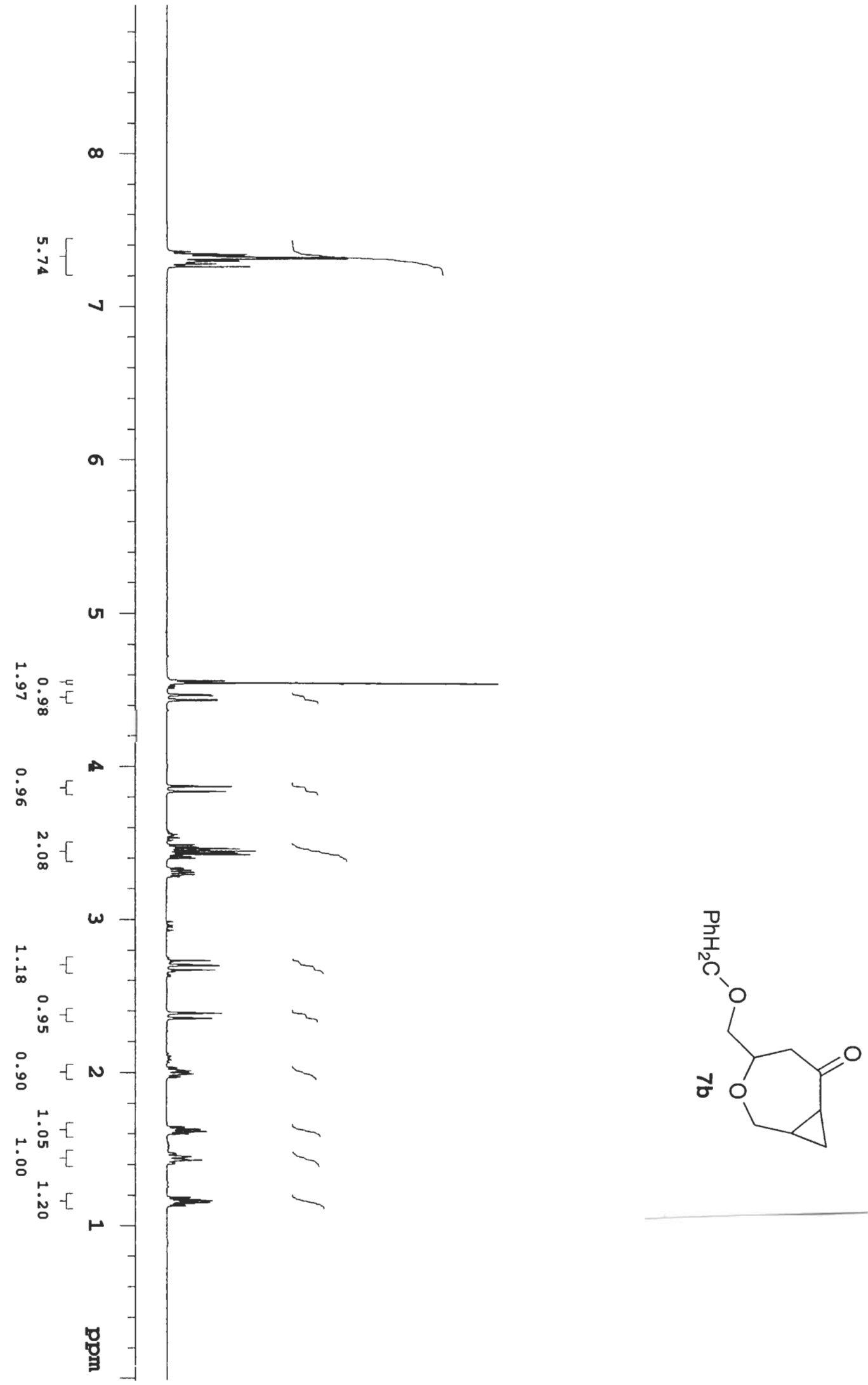

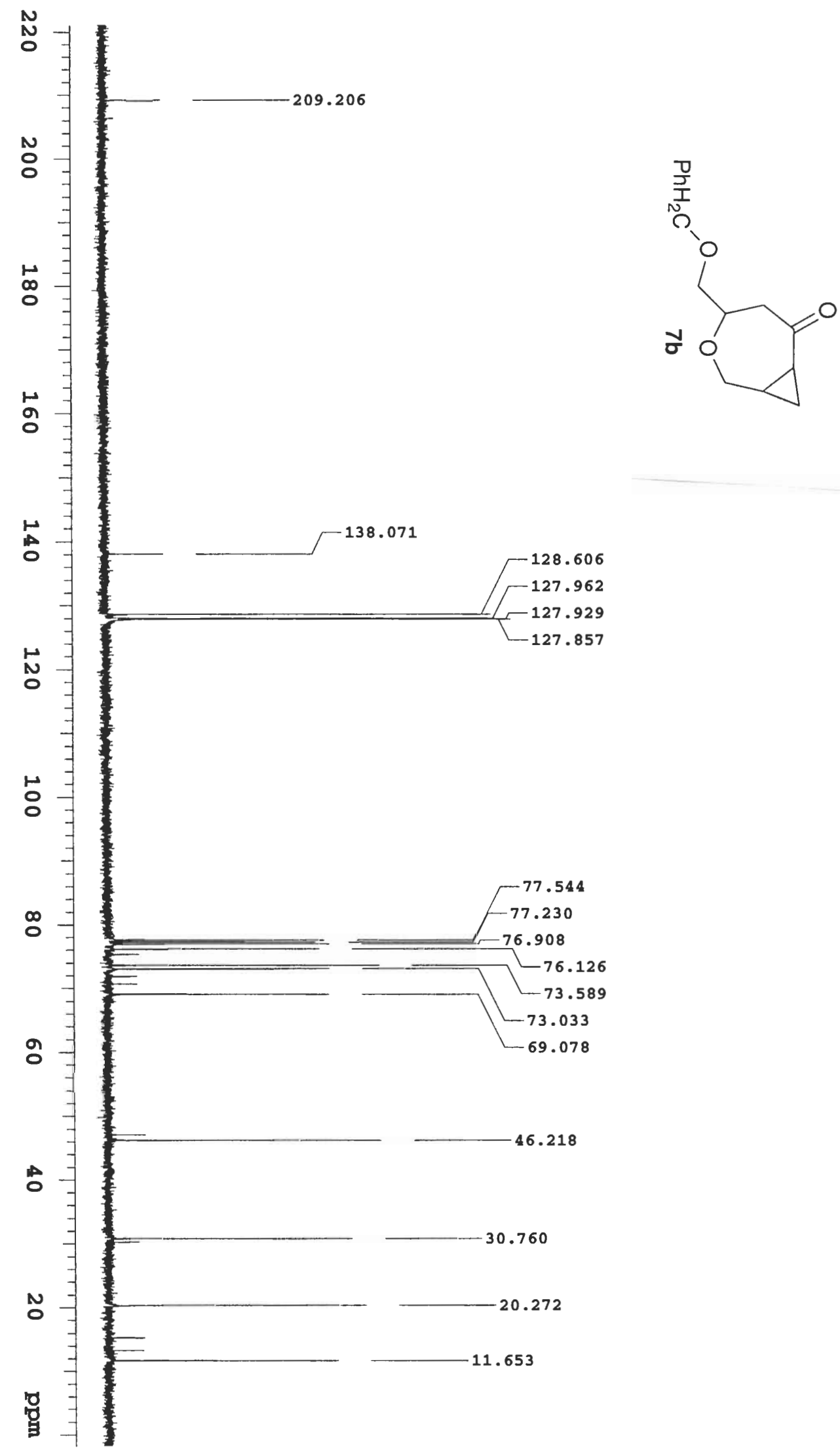

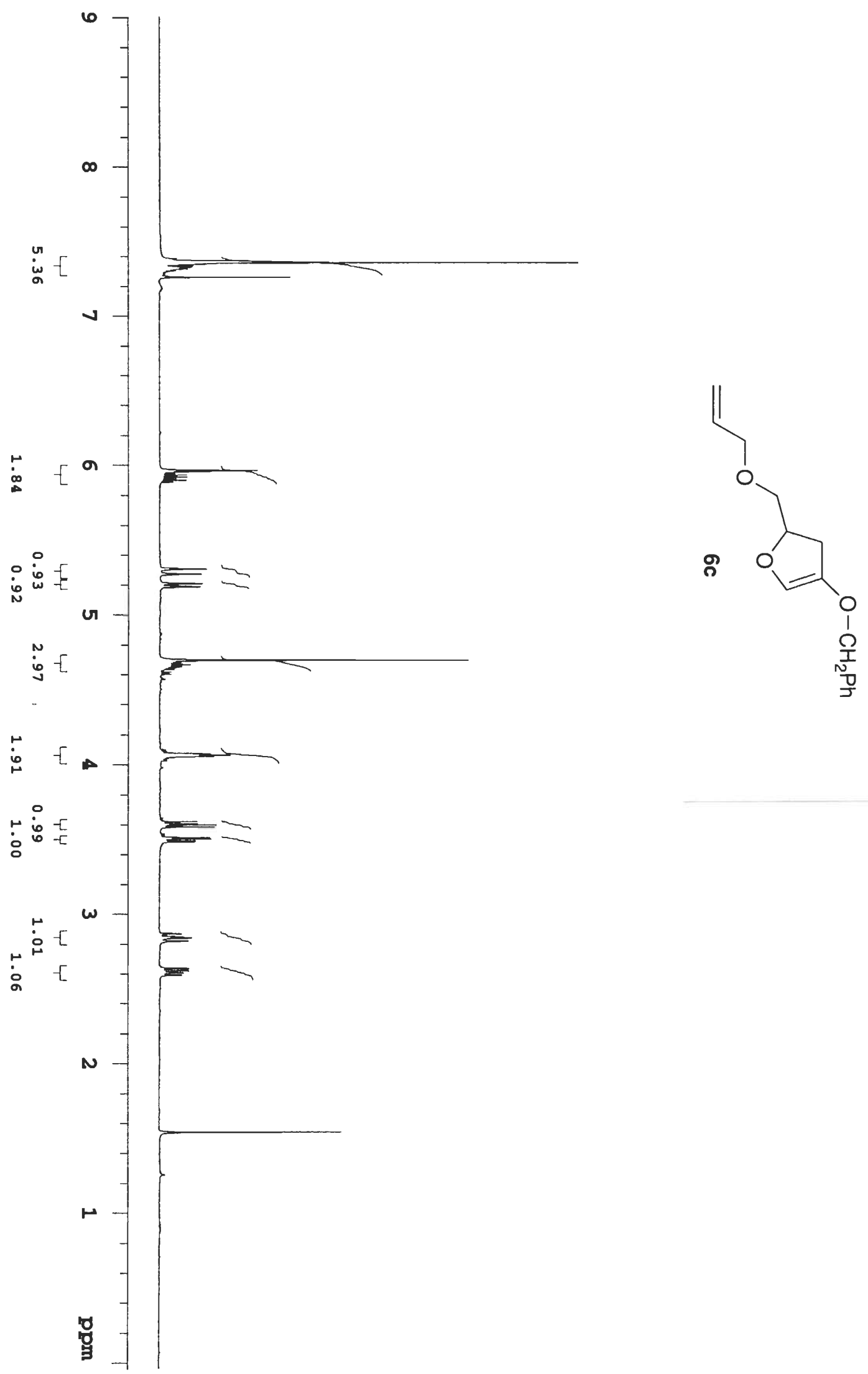


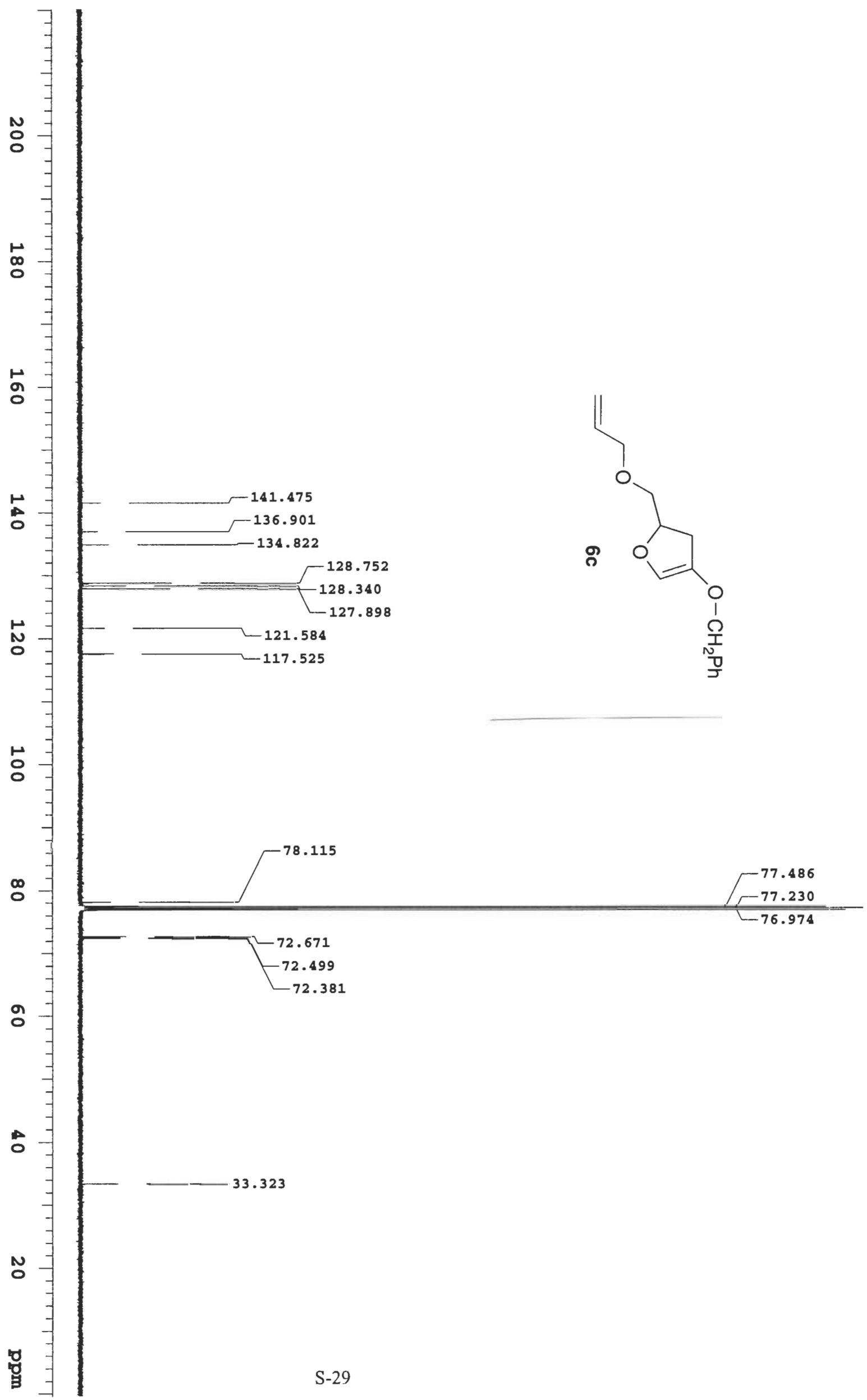



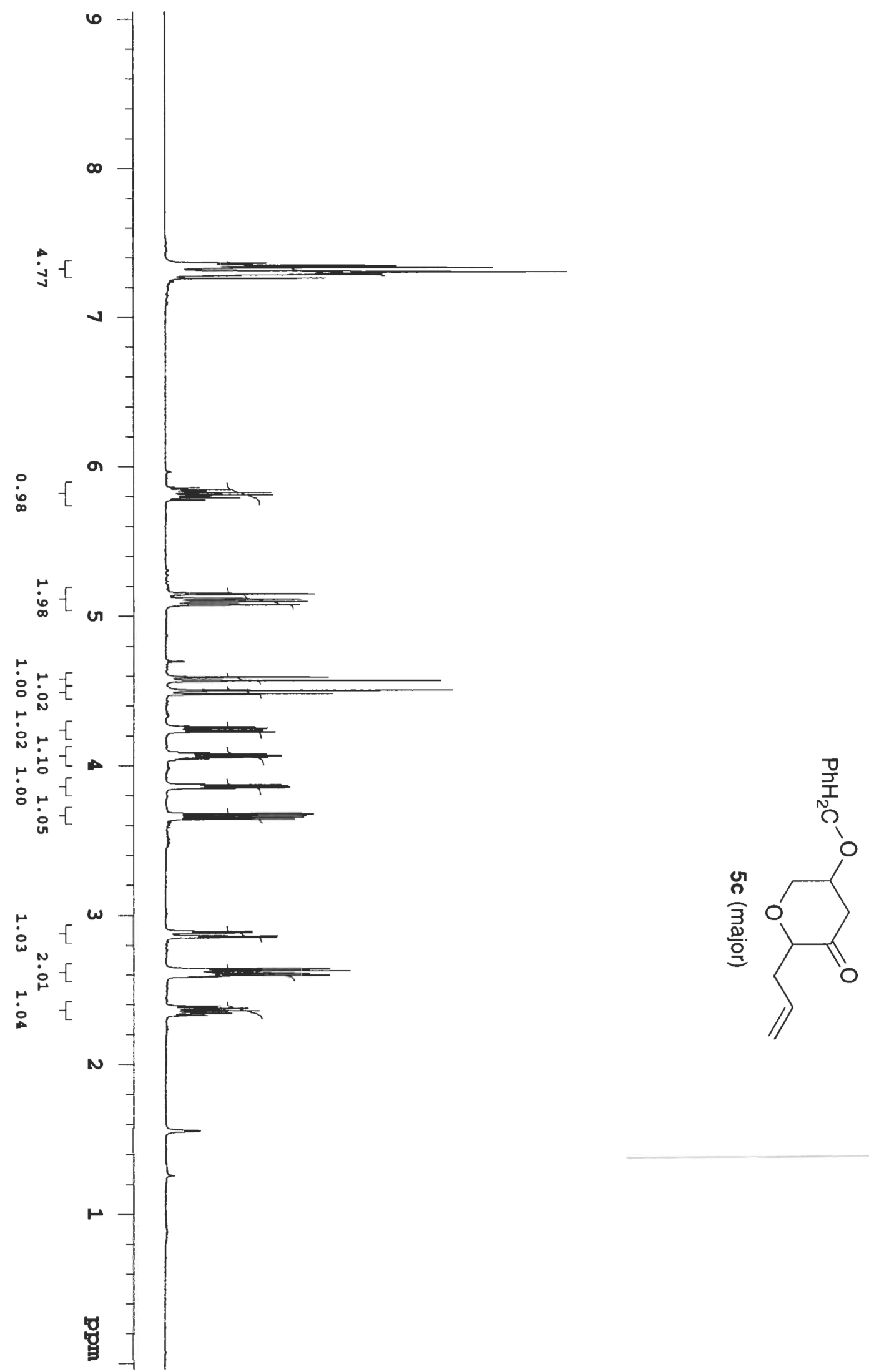


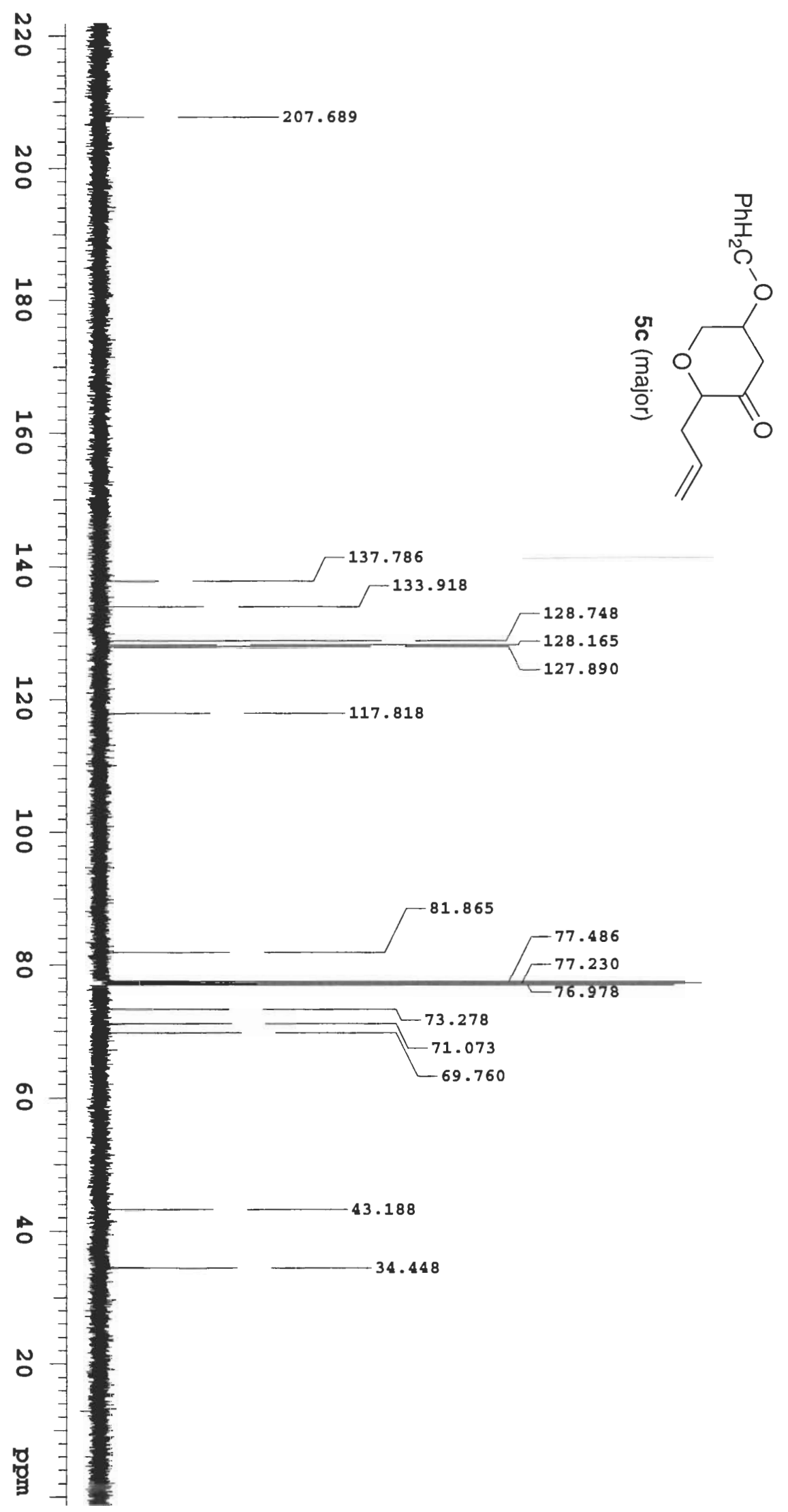




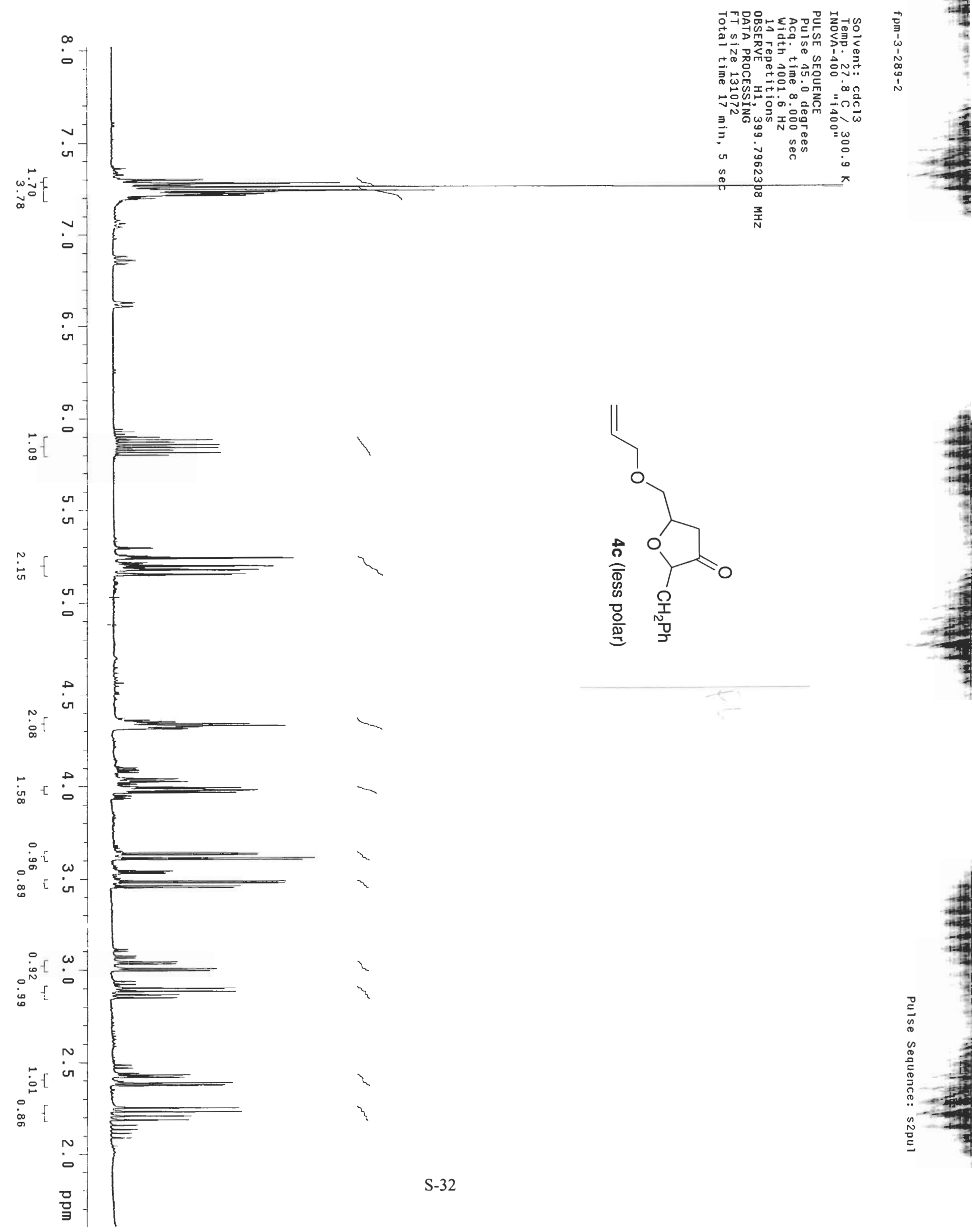




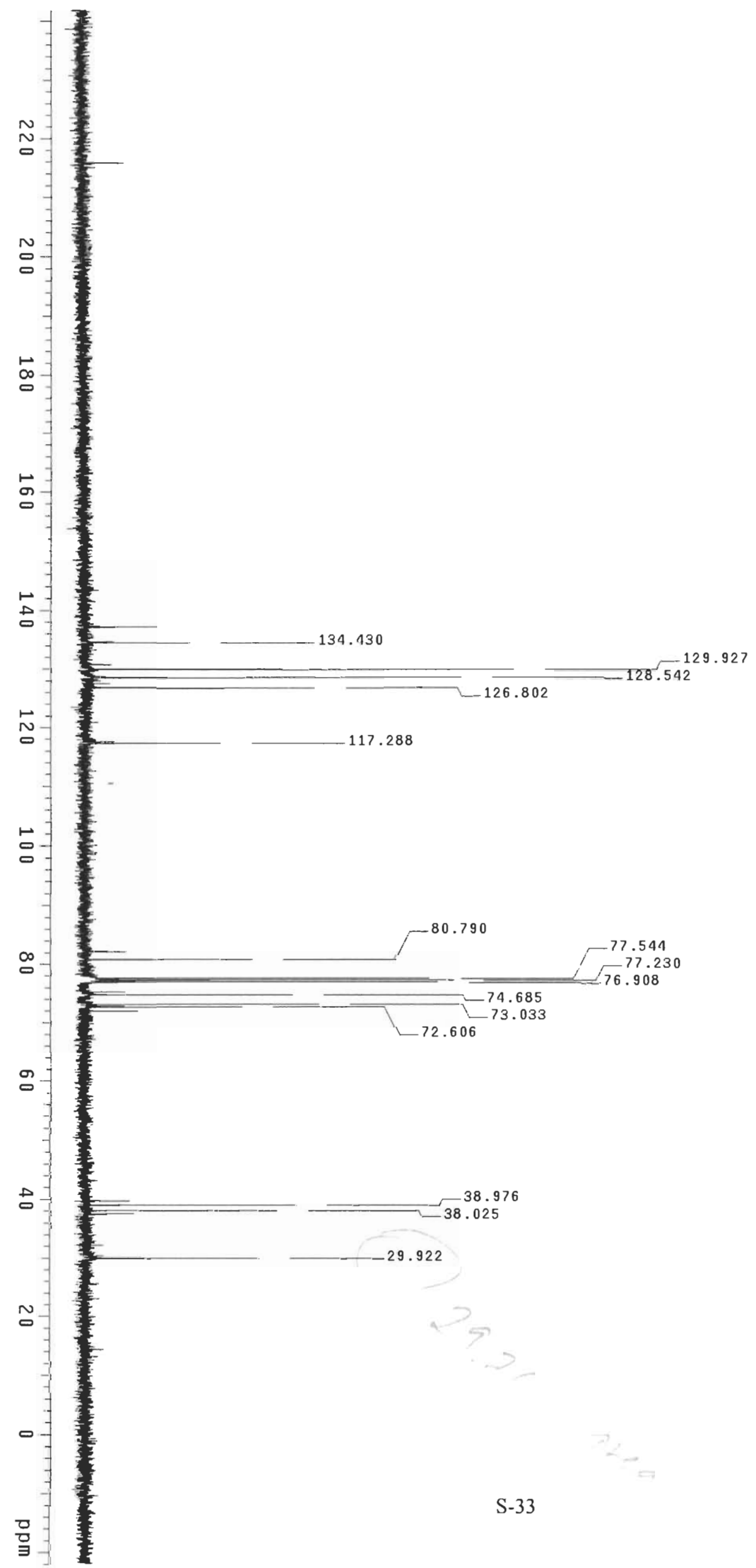

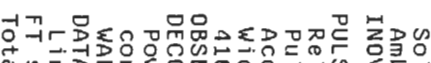

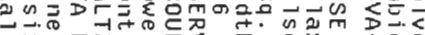
$+\infty$ 可

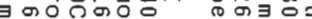

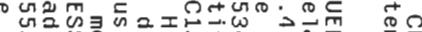

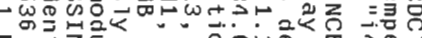

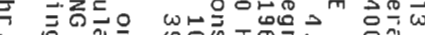

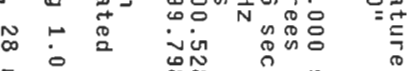

$\exists$

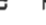

ज

ก

조N조N

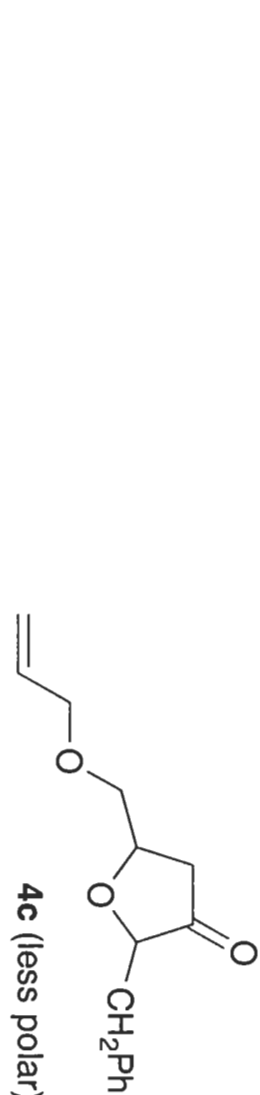




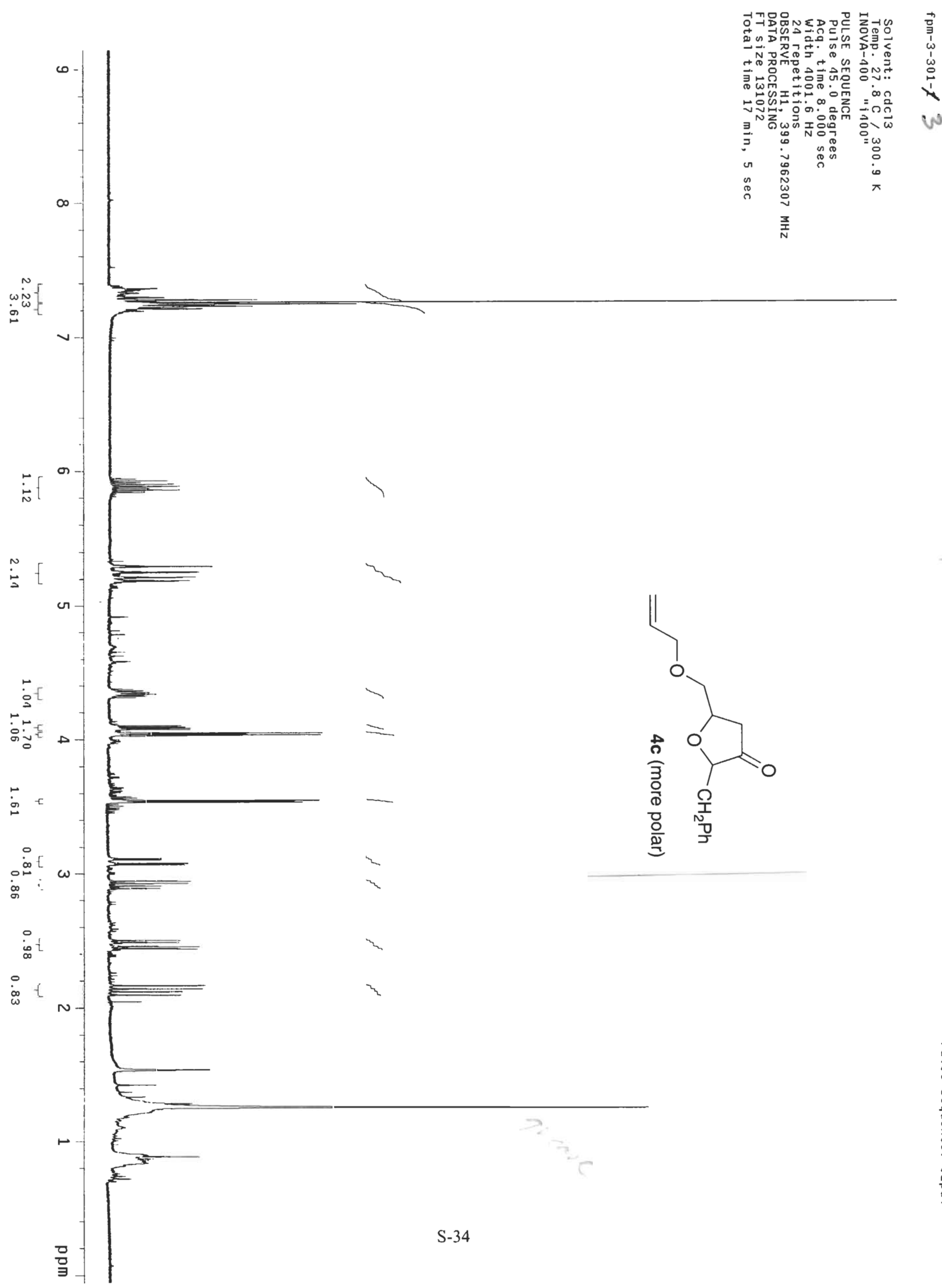



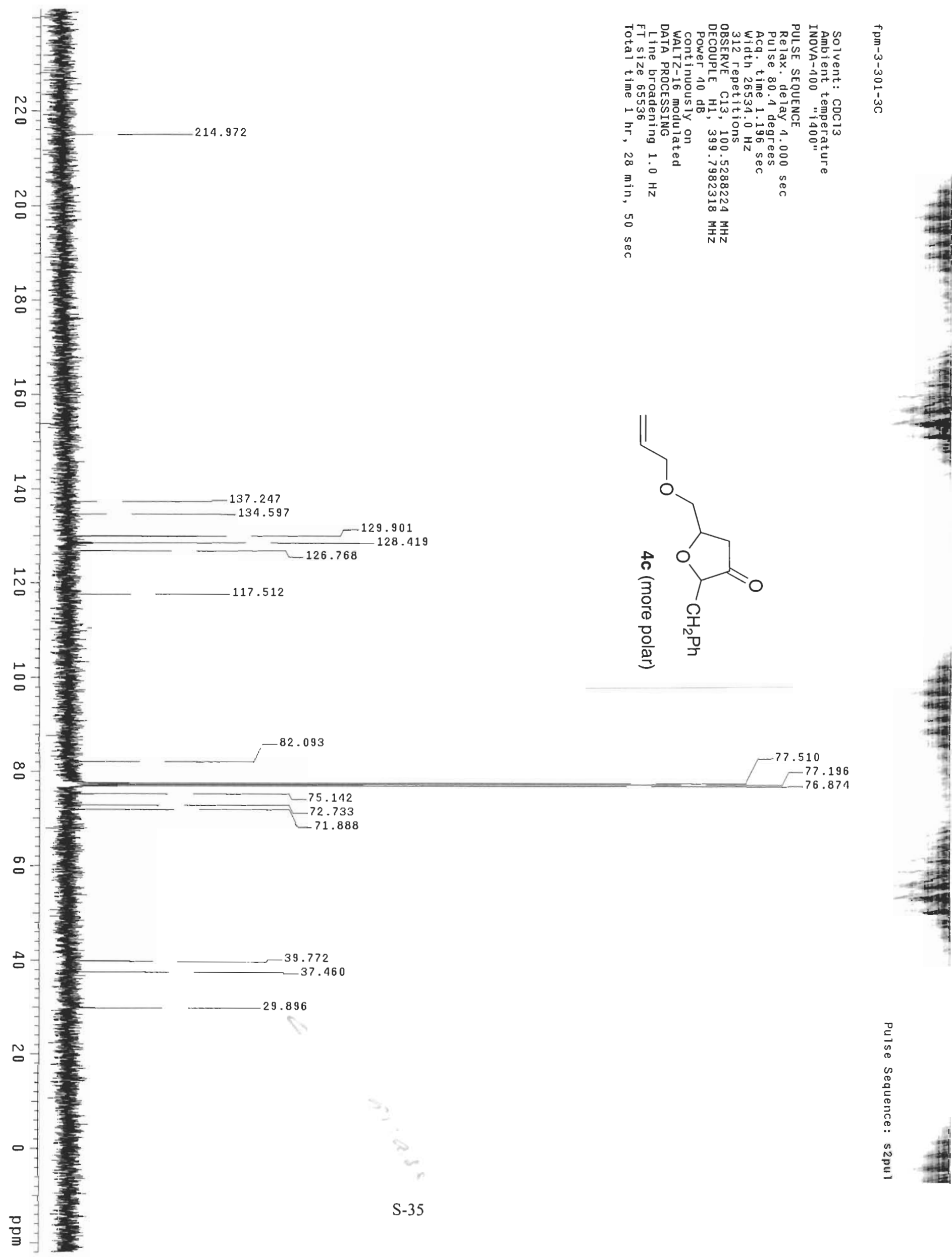

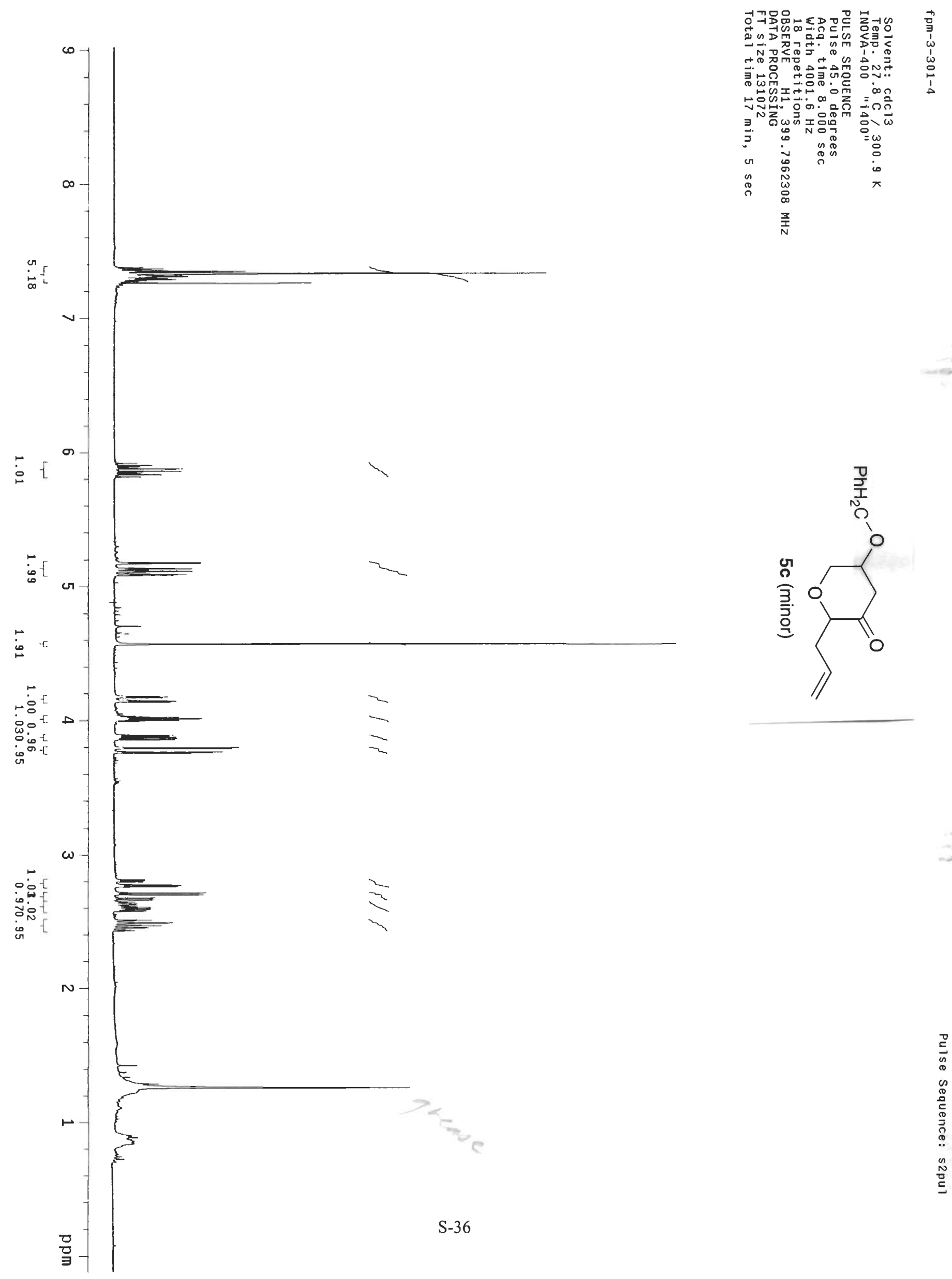

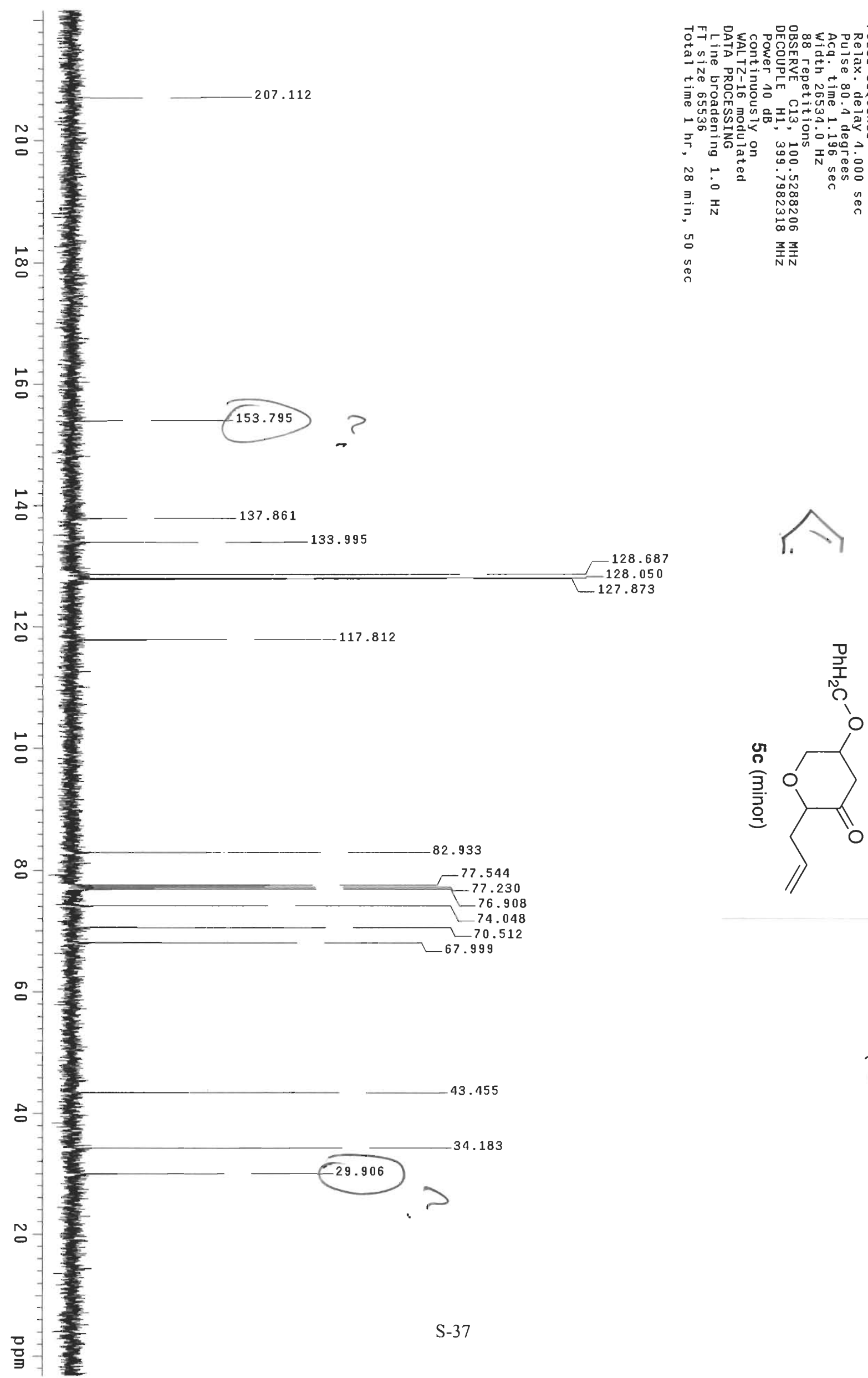

$\bar{\omega}$

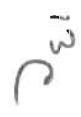

